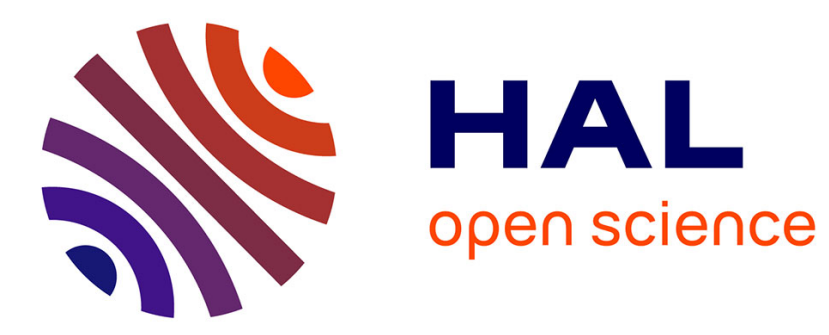

\title{
La Gascogne sous les premiers Plantagenêt (1152-1204)
}

Frédéric Boutoulle

\section{To cite this version:}

Frédéric Boutoulle. La Gascogne sous les premiers Plantagenêt (1152-1204). Martin Aurell; N.-Y. Tonnerre. Plantagenêts et Capétiens: confrontations et héritages, Colloque international des 13-15 mai 2004 organisé par les Universités de Poitiers et d'Angers, Brépols, pp.285-318, 2006, 10.1484/m.hifaeb.3.612 . hal-01793231

\section{HAL Id: hal-01793231 \\ https://hal.science/hal-01793231}

Submitted on 13 Oct 2020

HAL is a multi-disciplinary open access archive for the deposit and dissemination of scientific research documents, whether they are published or not. The documents may come from teaching and research institutions in France or abroad, or from public or private research centers.
L'archive ouverte pluridisciplinaire HAL, est destinée au dépôt et à la diffusion de documents scientifiques de niveau recherche, publiés ou non, émanant des établissements d'enseignement et de recherche français ou étrangers, des laboratoires publics ou privés. 
H I S T O I R E S D E F A M I L L E

LA PARENTÉ A U MOYEN ÂGE

Plantagenêts et Capétiens: confrontations

et héritages

Edité par Martin Aurell

et Noël-Yves TonNERRE 


\title{
La Gascogne sous les premiers Plantagenêts (1154-1199)
}

\author{
Frédéric Boutoulle.
}

Pour les possessions continentales des Plantagenêts, l'année de la mort d'Aliénor d'Aquitaine fut aussi celle du grand resserrement. Après la perte de la Normandie, de l'Anjou, du Maine et d'une grande partie de l'Aquitaine, le duché de Gascogne qui n'avait jusqu'alors été qu'une possession périphérique devint le principal fief continental des rois-ducs et cela jusqu'à la fin de la guerre de Cent ans. Or ce reliquat de l'orgueilleux «empire Plantagenêt * est aussi un des moins connus. Si l'on excepte les recherches anciennes de Pierre de Marca (1640), Jean de Jaurgain (1902) ou de Jacques Boussard (1956), peu de travaux se sont intéressés à la Gascogne Plantagenêt avant ce moment décisif. L'organisation du duché nous parait encore floue; l'action des premiers rois-ducs se révèle de manière lacunaire et sans ligne directrice; on ne sait pas vraiment pourquoi, lors de la crise de 12021204, la Gascogne leur est restée fidèle alors que, leur titulature en témoigne, les premiers Plantagenêts n'ont pas porté les titres gascons. Il faut donc chercher à savoir s'ils ont eu une politique particulière en Gascogne et comment ils ont imprimé leur autorité dans cet ensemble territorial.

Quoi qu'il eût été plus opportun d'intégrer le début du règne de Jean Sans Terre dans l'exposé, nous avons limité cette approche liminaire aux règnes d'Henri II (1154-1189) et de Richard $\mathrm{I}^{\mathrm{er}}(1189-1199)^{1}$. En effet, avant le règne de Jean et les premières séries de la chancellerie anglaise, la documentation permettant de percevoir l'action des rois-ducs en Gascogne est essentiellement extraite des cartulaires gascons et des chroniques, ce qui confère à cette période de 45 ans une homogénéité documentaire.

La politique du roi-duc vis-à-vis des puissants du duché s'y dessine assez bien comme les progrès des exigences ducale.

\section{Henri II et la Gascogne (1152-1170)}

\section{La Gascogne au début du règne d'Henri II}

Un territoire mal appréhendé par les contemporains

Les historiens rencontrent des difficultés pour délimiter précisément la Gascogne ducale au $\mathrm{XII}^{e}$ siècle, acquise par les comtes de Poitiers depuis le milieu du siècle précédent. Pour les contemporains, ces limites manquaient également de netteté car le nom recouvre au moins deux réalités, religieuse et ducale. La provin-

\footnotetext{
'Pour l'Agenais nous nous arréterons à 1196 quand ce diocèse passe sous la suzeraineté des comtes de Toulouse.
} 
cia Vasconia était l'ancienne Novempopulanie composée des onze diocèses de la province d'Auch (fig. 1) ${ }^{2}$. Le Comminges et le Couserans, quoi que faisant partie de la province, échappaient au duché de Gascogne depuis le XI ${ }^{\mathrm{e}}$ siècle ${ }^{3}$, mais si l'on en juge par la confusion entre province et ressort du sénéchal apparaissant rétrospectivement chez des Gascons du XIII siècle, cette distinction entre Gascogne ducale et Gascogne religieuse n'était pas intégrée de tous'. C'est à cette dernière Gascogne que pensait le poitevin Aimery Picaud: le Guide du Saint-Jacques de Compostelle, rédigé vers 1139, distinguait le Bordelais (Burdegalensium tellus) avec ses habitants (Burdegalenses rusticiores) et la Gasconia, peuplée de Gasconi, dans laquelle on ne rentrait qu'après l'éprouvante traversée des « Landes du Bordelais» ou, depuis Toulouse, après avoir franchi la Garonne ${ }^{5}$.

Cependant, au nord, le duché de Gascogne recouvrait les deux diocèses de la province ecclésiastique de Bordeaux, Bordelais et Agenais (jusqu'en 1196, pour ce dernier et le mariage de Jeanne avec le comte de Toulouse Raimond VI ${ }^{6}$. Aussi, quand Henri II et Aliénor séjournaient en Bordelais, à Saint-Macaire ou La SauveMajeure, ils reconnaissaient être "en Gascogne ${ }^{7}$; deux siècles plus tôt, Abbon de Fleury se rendant à la Réole par Poitiers et Angoulème avait considéré la Dordogne comme limite de la Gascogne ${ }^{8}$.

\footnotetext{
${ }^{2}$ P. de Marca, Histoine de Béam, Paris 1640, rééd. Pau, 2000, p. 309, d'après le cartulaire de Condom (1011) - apud provinciam Vasconiam Sancione illustri viri ducatum obtinente. Recueil des Historims des Gaules et de la France", (abrégé R.H.F.), éd. L. Delisle, Paris, 1879, t. XIII, p.108 (Guillaume de Neubourg, 1160), - Hi niminum olim $x$ Gasconia incerto auctore habentes originem, rgionibus plurimis virus suae perfidiae infude runt -; p. 294 (Robert du Mont, 1152), - pullulante perniciosa doctrina Henrici Haentici, maxime in Guasconia, suscitavit Dominus spiritum puellae junionis in illa provincia, ad hersim confutandam -; p. 658 (Otton de Freising. 1148, à propos de I'hérésie) - Iste in angulis Galliae, id est circa Britannam et Gasconima (co quod remotis ibi a conde Francia populis simplicitas vel potius, ut ita dixerim, stulticitas cui facile aror obrepere solet, abundat), verbum pracdicationis assumpserat -; R.H.F, t. XIV, p. 598, note b., Bernard de Clairvaux, - versus Tolosanas partes ad expugnandos henticos hacrsimque exstípandam ", s'arrête à Bordeaux (1145, également Gallia Christiana, t. II, col. 814) ; p. 639 (lettres de Pierre le Vénérable sur l'hérésie) - in provinia Novempopulana quae vulgo Gasconia vocatur ; R.H.F., t. XVII p. 704 (Mathieu Paris, 1208), « cira dies istos haerticorum pravitas, qui Albigrnses appellatur, in Wasconia, Arumpnia et Albigesi in partibus Tolosanis et Arragonum ngno -. La province de Novempopulanie comprend les diocèses d'Aire, Auch, Bayonne, Bazas, Dax, Lectoure, Lescar, Oloron, Saint-Bertrand de Comminges, Saint-Lizier de Couserans et Tarbes.

${ }^{3}$ R.H.F., (voir note 2) t. XIX, p. 69, (Pierre des Vaux de Cernay, 1212) : les évêques de Comminges et de Couserans demandent à Simon de Montfort que - sine pugna et gladio maximam mapert Wasconiae partem. -

'Petit cartulaire de La Sauve-Majeure, Bibliothèque municipale de Bordeaux, ms 770 (1237), - tempore predictorum ngum, Hennici at Ricardi, quod unicus aset smoscalcus totius tere rgis in archippiscopatum Burdega-
lensis at Auxitano, .

${ }^{5}$ Le guide du pèterin de Saint-Jacques de Compostelle, éd. J. Vieillard, Mâcon, 1959, p. 16-20. Dans le Guide, tellus est utilisé au sens d'unités politiques (royaume, comté ou duché), linguistiques et ethniques (* tellus Pictavorum, tellus Sanctonensium, tellus Gasconia, Navarrorum tellus, tellus Pictavorum, tellus Basclorum $)$ ).

${ }^{6}$ R.H.F., (voir note 2) t. XIX. p. 62 (Pierre des Vaux du Cernay), - Erat enim Agennum civitas nobilis inter Tolosam at Burdegalam... Civitas illa cum territorio suo a dirbus antiquis fuerat ngis Anglie; sed Ricardus quando dedit sororm suam Johannam Raimundo comiti Tolosano in uxorrm, civitatem sarpedictam cum territorio suo dedit pro matrimonio sororis suae comiti Tolosano. -

"Recuril des actes d'Henri II, roi d'Angletere duc de Normandie concernant les provinces françaises at les affaims françaises, éd. L. Delisle Paris, 1916, t. I., p. 432-433; R.H.F., (voir note 2) t. XVI p. 346-347; E. du Laura, Histoine de l'abbaye de La Selve Maiour, 1680-1683, Bibl. municipale de Bordeaux, Ms 1870, p. 165, éd. J. F. Duclot, J. F. Larché et J. C. Tillier, Camiac-et-Saint-Denis, 2003, p. 121.

"Aimoin, Vita s. Abbonis abbatis Floriacensi, Patrologie latine, éd. P. Migne, t. 139, col. 409-411, - Inde ad Dondoniam fluenta ventum: quo enavigato amne, Gasconiae fines ingrediuntur. -
} 
Désaffection des titres de comte et duc de Gascogne chez les Plantagenêts

Cette région périphérique et mal appréhendée n'était pas non plus valorisée par la titulature des premiers Plantagenêts. Alors que leur prédécesseur Guillaume VIII (1040/1063-1086) s'était fait connaître comme « duc de Gascogne et comte de Gascogne ${ }^{9}$, le titre de comte de Gascogne n'apparait qu'une seule fois à notre connaissance associé à Henri II, dans la date d'un acte du cartulaire de Sainte-Croix de Bordeaux daté de $1174^{10}$. Quant à Richard, les actes du Bordelais et du Bazadais le présentent comme " comte de Poitiers * ou a duc d'Aquitaine *

On peut d'abord interpréter cette désaffection par l'ancienneté de l'union de la Gascogne à l'Aquitaine. Du reste, les Gascons eux-mêmes avaient fini par «oublier* que les prédécesseurs d'Henri et de Richard avaient été comtes et ducs de Gascogne: des textes rédigés dans les cartulaires de Sainte-Croix de Bordeaux et de Saint-Seurin de Bordeaux dans la seconde moitié du XII ${ }^{e}$ siècle attribuent aux comtes de Poitiers des donations faites autrefois par les comtes de Gascogne ${ }^{12}$,

Mais cette indifférence pour les vieux titres gascons et leur éclipse par les titres poitevins est, semble-t-il, davantage la conséquence d'une redéfinition de l'aire de domination ducale entre le milieu du $\mathrm{XI}^{e}$ siècle et le milieu du XII ${ }^{e}$ siècle. En effet, au $\mathrm{XI}^{e}$ siècle le comté de Gascogne offrait à son titulaire la domination sur la * Gascogne majorale ^, un ensemble s'étendant du Bazadais à la Lomagne au nord et sur un bloc occidental formé des diocèses de Labourd, Lescar, Dax et Aire (fig. 1) ${ }^{13}$. Le titre ducal, associé au comté de Bordeaux, apportait une suzeraineté sur les comtés mineurs de Fezensac-Armagnac, Astarac, ainsi que sur le comté de Bigorre ${ }^{14}$. Or les successeurs de Guillaume VIII, les ducs Guillaume IX et Guillaume X, ont vu leur aire d'action se réduire considérablement ${ }^{15}$. Faute d'intervenir dans les anciens comtés mineurs, mais également en Béarn après Guillaume VIII où l'essentiel des prérogatives publiques avait été abandonné au vicomte, les titres de duc

\footnotetext{
" - Le cartulaire du prieuré Saint-Pierre de La Réole ", Anchives Historiques du département de la Gironde, éd. Grelet-Bargerie, t. V, Bordeaux, 1863 (abrégé en cart. La Réole), n43 (1070), - ad comitem Guillelmum, ducem Vasconie, proclamationis causa, in castro Taicavad =; $\mathbf{n}^{\circ} 60$ (1086), = actum in cenobio Regule qui dicitur Squin, mgnante Philippo mge Francorum, primo anno Guillelmi Gasconum comitis, ac primo anno vicecomitis Bernandi Bralmes ; Cartulaire de l'abbaye Sainte-Croix de Bordeaux, Anchives Historiques du département de la Gironde, éd. M. Ducaunnès-Duval et L. Drouyn, t. XXVII, Bordeaux, 1892165 (abrégé en cart. Sté Croix), n*105 (1138), = Guillelmus Helias primo anno quo Lodovicus rax Franconum duxit in matrimonium filiam comitis Pictavorum at Vasconum. *

${ }^{10}$ Cart. Ste-Croix, n*11 (1174), - Henrico Anglorum nge, Aquitanie duce, Vasconie comite. -

"Cependant, dans la confirmation des privilèges de l'abbaye de Saint-Sever, Richard précise * in toto comitatu nostro=: P. D. Du Buisson, Historia monasterii Sancti Severii libri X, éd. J. F. Pédegert, A. Lugat. 1876. p. 200. - L'amour a la guerre s, édition critique de l'autur de Bertran de Born, éd. G. Gouiran, Aix-enProvence, 1985 (sirventes $n^{\circ} 11$ ) : dans le sirventes $D^{\prime}$ 'un sirventes no-m cal far loignar gands, Bertrand de Born présente des titres que Richard ne met pas en avant dans sa titulatur, = Ni sera ducs de la terra normand ni comes palats ni de Bondels ni dels Gascos part Landa, seigneur ni de Basats. -

${ }^{12}$ Dans un contentieux opposant l'abbé de Sainte-Croix de Bordeaux et le dominus d'Agassac, les moines prétendent tenir la villa de Macau, - de donatione domini videlicet bone memorie olim comitis Pictavensis et successorum ipsius = (cart. Ste-Croix, n70, 1187-1197). Dans un autre contentieux opposant les chanoines de St-Seurin à Arnaud d'Illac, les premiers assurent tenir des landes a a donatione Sanctii, comitis Pictavensis =: Le cartulaire de l'oglise collégiale Saint-Seurin de Bordeaux, éd. J. A. Brutails, Bordeaux, 1897, n 165 (abrégé en cart. St-Srurin), (1199).

${ }^{13}$ R. Mussot-Goulard, Les Princes de Gascogne, Marsolan, 1982, p. 125-130. Pour la localisation des seigneuries et des localités, nous prions de lecteur de se reporter aux cartes (figures 1 et 2).

$"$ R. Mussot-Goulard, (voir note 13), p. 16-117.

is Ibidem, p. 209-215.
} 
ou de comte de Gascogne ne correspondaient plus à la domination effective des Poitevins. Nous ne savons malheureusement pas si cet abandon de la titulature gasconne a accompagné des accords avec l'aristocratie bénéficiaire de ce recul de la puissance ducale ou s'il s'agit d'une reconnaissance de fait.

\section{Recul de la suzeraineté ducale au sud}

Au milieu du $\mathrm{XII}^{e}$ siècle, le recul de la suzeraineté ducale au sud du duché de Gascogne profitait essentiellement au roi d'Aragon occupé à rassembler des territoires sur les deux versants des Pyrénées. En 1122, le comte de Bigorre Centulle II avait prêté hommage à son compagnon d'armes Alphonse $\mathrm{I}^{\text {er }}$ Le Batailleur (11041134) pour le comté de Bigorre et tout ce qu'il devait acquérir ${ }^{16}$. Or, vers 1125 , la fille et héritière de Centulle II, Béatrix, épousa Pierre II vicomte de Marsan (vers 1125-1163): dès lors Pierre porta les deux titres (fig. 1) ${ }^{17}$. Leur fils Centulle III (1161-1185), comte de Bigorre et vicomte de Marsan, suivit les pas d'Alphonse II d'Aragon (1162-1196) et reçut des dons de lui ${ }^{18}$. En septembre 1192, Alphonse II d'Aragon fiançait sa pupille Pétronille, comtesse de Bigorre et vicomtesse de Marsan, au vicomte de Béarn Gaston VI et donnait à celui-ci le comté de Bigorre contre l'hommage et la fidélité ${ }^{19}$.

Le vicomte de Béarn suivait en effet la même voie. En avril 1154, à Canfranc, une délégation de 120 béarnais conduite par les évêques de Lescar et d'Oloron fit de Raimond Béranger IV, comte de Barcelone et époux de l'héritière d'Aragon, le tuteur du jeune vicomte, Gaston V, fils de Pierre III de Gabardan-Béarn, et lui prêta hommage (hominium sacramenta) ${ }^{20}$. Or depuis le $\mathrm{XI}^{\mathrm{e}}$ siècle, les vicomtes de Béarn étaient aussi vicomtes d'Oloron, de Montaner et possédaient les deux vicomtés septentrionales du Gabardan et du Brulhois (fig. 1). Le 30 avril 1170, Marie, scur du défunt Gaston V, prêtait hommage au roi d'Aragon Alphonse II à Jaca non seulement pour le Béarn, mais aussi pour le Gabardan et le Brulhois pourtant situées au nord de l'Adour ${ }^{21}$. Alphonse II lui donna pour époux Guillaume de Montcade, ricombre d'Aragon, duquel il reçut l'hommage pour la vicomté de Béarn en mars 1171, à Saragosse.

Plus à l'ouest, le roi d'Aragon avait posé d'autres jalons. Le Guide du pèlerin de Saint-Jacques reproche au roi d'Aragon et à certains seigneurs (Raimond de Solis Soule?- Vivien d'Aigremont, Arnaud de la Guigne et le vicomte de Saint-Michel) d'être de connivence et de se partager les extorsions levées par les péagers sur les pèlerins empruntant la route des ports de Cize vers Roncevaux, entre Ostabat,

\footnotetext{
${ }^{16} \mathrm{~J}$. de Jaurgain, La Vasconie, ćtude histonique et critique, deuxième partie, Pau, 1902, t. II, p. 382. Centulle II et son cousin Gaston IV de Béarn ont participé à la prise de Saragosse en 1118 . En janvier 1128 il épouse en seconde noces Stéphanie de Barcelone, fille de Raimond Béranger III comte de Barcelone. $17 \mathrm{~J}$. de Jaurgain, (voir note 16) p 382: en 1130, Pierre de Marsan est comte de Bigorre et seigneur de Tarazona.

is Ibidem, p. 387 : le val d'Aran.

${ }^{19}$ P. de Marca, (voir note 2) p. 212 et J. de Jaurgain, (voir note 16) p. 389 : - commendo et dono tibi Gaston, nobili vicecomiti Bearnensi, totum comitatum meum et terram de Bigorra, simul cum dilecta consaguinea mea, filia dilecti nostri Bernardi nobilis comitis de Comenge, nepte Centulli-. Pétronille meurt en 1251 après avoir été
mariée cinq fois. mariée cinq fois.

э. P. Tucoo-Chala, La vicomté de Bíarn et le problème de sa sourcraineté des origines à 1620, Bordeaux, 1961, p. 42, et doc. 5 p. 147. J.-P. Barraqué, -Béarn et Aragon aux $\mathrm{XI}^{*}$ et XII' siècles-, dans Aquitaine Espagne (IIIr-XIII siècle), textes réunis par Ph. Sénac, Civilisation médiévale XII, CESCM, Poitiers, 2001, p, 175-188.

${ }^{21} \mathrm{Ibidem}$, p. 44-45 et doc. 6 p. 148 ; Marie donne d'ailleurs le castrum de Gabarret, en Gabardan et celui de Manciet.
} 
Saint-Jean et Saint-Michel-Pied-de-Port (fig. 1) ${ }^{22}$. Cet intéressement du roi d'Aragon s'explique certainement par l'ancienne union avec la Navarre, pourtant révolue après $1134^{23}$. Aussi la suzeraineté sur le Béarn offrit-elle à l'Aragon un nouveau moyen d'action sur ce secteur nord-pyrénéen car depuis l'acquisition de la vicomté d'Oloron les vicomtes de Béarn n'ont jamais cessé de lutter contre les vicomtes de Dax et les vicomtes de Soule pour contrôler la confluence des Gaves ${ }^{24}$.

Le tronçon de la voie, au sud de Sorde, entre Ostabat et Saint-Jean-Pied-de-Port constituait donc la limite occidentale de l'influence aragonaise au debut de la seconde moitié du $\mathrm{XII}^{e}$ siècle. Plus à l'ouest en effet, d'après l'Histoire de Vézelay d'Hugues de Poitiers, - le Pays Basque (Basclonia), la Navarre jusqu'aux Pyrénées [cispyrénéenne] et jusqu'à la Croix de Charles [au sommet du col de Roncevaux] ont été apportées à Henri II par Aliénor d'Aquitaine ${ }^{25}$. Ainsi, le Labourd, avec les vallées de Baigorry, Ossès, Cize, Irissary, Ilhody et Armendarits, qui avaient pourtant fait partie du royaume de Pampelune jusque vers 1120, relevaient du duc d'Aquitaine ${ }^{26}$.

\section{Les actions d'Henri II en Gascogne}

\section{Les premières apparitions (1156-1166)}

La première apparition d'Henri II en Gascogne, datée de décembre 1156, lui permit d'emblée d'affirmer sa position. Accompagné d'Aliénor, Henri séjourna en Bordelais; le 13 décembre ils étaient à La Sauve-Majeure pour confirmer les privilèges de l'abbaye et placer le monastère sous protection royale ${ }^{27}$; neuf jours plus tard, Alinéor renouvelait la confirmation ${ }^{28}$. A Noël, Henri réunit à Bordeaux les

$z_{z}$ Ed. J. Vieillard, (voir note 5) p. 22, - Quapropter prrcipimus et exoramus ut hi portageri et nex Aragonensis ceterisque divites qui tribuit peccunias ab ris accipiunt omnesque illorum consencientes, scilicet Raimundus de Solis at Vivianus de Acromonte et vicocomes de Sancto Michaeles.

${ }^{2}$ L'auteur du Guide ignore en effet la séparation de la Navarre et de l'Aragon, effective depuis le début du règne de Garsie Ramirez IV: ainsi la Navare n'est pas citée parmi les trois pays que le pèlerin voit en arrivant au sommet du Port de Cize (Castille, Aragon et France) : éd. J. Vieillard, (voir note 5 ) p. 25. Cette union avait permis à Alphonse $\mathrm{I}^{*}$ le Batailleur de faire une charte depuis le château de Bayonne le 26 octobre 1130: J. de Jaurgain, (voir note 16) t. II, p. 382.

${ }^{24} \mathrm{~J}$. de Jaurgain, (voir note 16) t. II, p. 462-467; F. Boutoulle, - Le conflit béarno-dacquois et les croisades de 1149 , Bulletin de la Socicté de Bonda, n*479, $3^{*}$ trimestre 2005, p. 341-356.

s R.H.F., (voir note 2) t. XII, p. 341 ; J. de Jaurgain, (voir note 16) t. I., p. 231 : parmi les pays apportés par Aliénor à Henri II, * Aquitania, Gasconia, Basclonia et Navarriam usque ad Pyrenacos montes at usque ad Crucem Caroli. = On note que selon ce témoignage le pays Basque n'est pas considéré comme partie intégrante de la Gascogne. La Croix de Charles est également mentionnée dans les limites du diocèse de Labourd, passant pour avoir été fixées en 980, ainsi que dans le Guide du Pilerin.

๙ J. de Jaurgain, (voir note 16) t. I, 222-235 et t. II p. 230. Présence des vicomtes de Labourd, Arberoue et Soule à Sorde, en 1120 près de Guillaume IX.

${ }^{2}$ A.D 33, H 12 f. 1 (vidimus de 1281-1282); Ed. L. Delisle, (voir note 7) t. 1., p. 118 ; Grand cartulaire de La Sauve Majeur, 2 t., C. et A. Higounet, Fédération Historique du Sud-Ouest, Bordeaux, 1996 (abrégé en G.C.S.M.), n"1109, confirmation de Richard de l'acte de son père: - precipimus ctiam prepositis $t$ ministris nostris ut ubicumque res predicte colesie fuerint, pro posse suo custodiant et defendant ut nec illis injuriam aliquam vel violentiam inferant. =

s E. du Laura, (voir note 7) p.119 et 233; A.D. 33, H. 266, f 61 (analyse moderne). Henri confirme peut-être lors de ce séjour bordelais au monastère de Maillezais la possession de l'église Saint-Martin du Mont-Judaique près de Bordeaux: A. Richard, Histoine des comtes du Poitou, Paris, 2 t., 1903, p. 123; Abbé Lacurie, Histoine de Mailleais depuis sa fondation jusqu'à nos jours, Fontenay-le-comte, 1852, p. 257 258 (I'acte de confirmation par le roi depuis Bordeaux n'est pas daté). 
«barons de Gascogne * et d'autres «gens du peuple " pour dicter une paix sur laquelle on ne sait rien ${ }^{29}$. En cette occasion, d'après Gervais de Canterbury, Henri reçut les $\approx$ hommages et des otages de la Gascogne ${ }^{30}$, à l'instar de Louis VII lors de son mariage avec Aliénor à Bordeaux, le 25 juillet $1137^{31}$. En janvier 1159, Henri II et Aliénor rencontraient à Blaye, au nord du Bordelais, le comte de Barcelone, Raimond Bérenger IV, pour contracter une alliance contre le comte de Toulouse ${ }^{32}$. Cette année là (janvier ou février) Henri II tenta d'imposer son candidat à l'archevêché de Bordeaux, l'écolâtre de Poitiers Jean de Sie, et, après un premier échec, put installer le doyen de la cathédrale du Mans, Hardouin (23 décembre 1159) ${ }^{33}$. L'ost qu'il conduisit vers Toulouse en 1159, in fines Gasconie, comprenait des Gascons malheureusement anonymes ${ }^{34}$. Inconnus également les représentants du baronnage gascon qui assistaient à l'assemblée du Mans où Henri II, le 11 mai 1166, décréta une imposition générale dans ses états pour participer à la défense de la

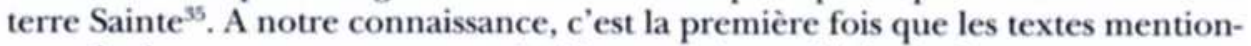
nent des barons gascons en cour du Plantagenêt aussi loin de chez eux.

\section{La répression de la révolte du baronnage aquitano-gascon (1169-1170)}

Cependant, le soulèvement de 1168-1169 ébranla la fidélité du baronnage gas$\operatorname{con}^{36}$. Henri II dut descendre en Gascogne entre Carême et le 15 août 1169. Selon Robert du Mont, il y détruisit de nombreux castella (anonymes) fortifiés contre $\mathrm{lui}^{37}$.

${ }^{2}$ R.H.F., (voir note 2) t. XII, p. 121 (1156, Chronique de l'anonyme), - Pace itaque inter populum celebrata [à propos de Limoges ], adiit Burdegalam; ibique Natalis Domini fostivitate celebrata, Baronibus Vasconie at aliis populis pacem indixit. Deinde reversus Angliam, panvo tempore fono at armis Galenses sibi substravit. =; R.H.F., (voir note 2) t. XII, p. 417 (1156, Richard le Poitevin) : * Pace itaque inter populum celebrata, consilio domini Gaufredi tunc temponis Bundegalensis archiepiscopi, primique aedificatoris Ecclesie Sabluncellensis adiit Burdegalam; ibique Natalis Domini festivitate celebrata, baronibus Vasconie at aliis populis pacem indixit. Deinde ruersus Angliam, parvo tempore ferwo et armis Galenses sibi substravit. -

so R.H.F., (voir note 2) t. XIII, p. 126, - hominia $t$ obsides de tota Aquitanie et Gasconia argit et accepit " (1156).

${ }^{31}$ R.H.F., (voir note 2 ) t. XII, p. 84.

s R.H.F., (voir note 2) t. XIII, p. 302 (Robert du Mont).

ss R.H.F., (voir note 2) t. XII, p. 399 (Historia Pontificum at comitum Engolismensis), - Qui cum de dectione secreto tractarent, Henricus nex se intermiscuit concilio eorum, deprecans, quatinus Johannes de Saecli magister scolarum Pictauensium per eos in archiepiscopum eligertur ; O. Pontal, - Les évêques dans le monde Plantegenèt =, Cahiers de civilisation médiévale, t. XXIX, 1986, p. 129-137.

${ }^{3}$ R.H.F., (voir note 2) t. XIII, p. 127 (Gervais de Canterbury, 1159), descente contre Toulouse avec - omnes comites atque barones Angliae at Normaniae, Aquitaniae, Andegaviae et Guasconiae at multi aliii ex diversis mgionibus milites et pedites ad eum venidbant. =; R.H.F., t. XIII, p. 105 (Guillaume de Neubourg), - Memorabilis nx Anglorum Henricus secundus anno mgni sui septimo in partes Gasconie duxit exercitum; cujus expeditionis causa erat. Comes Pictaviensis (...) Rex Anglorum contracto ex universis ternis suae ditionis exercitu, Gasconiae fines ingresus est. =

ss R.H.F, (voir note 2) t. XIII, p. 128 (11 mai 1166, charte d'Henri II au Mans) : A. Richard, (voir note 28) p. 140. Pour fournir des subsides aux défenseurs de la Terre Sainte, Henri décrète en assemblée solennelle une taxation générale des particuliers inspirée des impositions de paix, en présence de trois archevêques, onze évêques - et multis barones mei de Normannia, de Cenomannia, de Turona, de Adegavia, de Britannia, de Guasconia. .

${ }^{36}$ Dans l'intervalle, en 1162, - Henricus $m x$, ordinatis et compositis nbus et castellis suis in Normannia, Aquitania, Andegavia et ctiam in Wasconia =, R.H.F., (voir note 2) t. XIII, p. 307 (Robert du Mont)

37 R.H.F., (voir note 2) t. XIII, p. 313 (Robert du Mont,1169), - Rox Henricus perrexit in Quadragesima Wasconiam $a$ destructis multis castellis que contra cum erant vel munitis (...). Mense augusto pacificatis fere omnibus in Pictaxensi pago, Wasconia Henrici nx venit in Normanniam. - 
Saint-Macaire doit être un de ceux-là. A la limite du Bordelais et du Bazadais, sur la rive droite de la Garonne, Saint-Macaire était un site de première importance avec son castrum et son prieuré bénédictin dépendant de l'abbaye Sainte-Croix de Bordeaux (fig. 1). Le roi était en effet à Saint-Macaire in Gasconia, en 1169, d'où il adressa deux lettres relatives à ses démêlés avec Thomas Becket ${ }^{38}$. On peut d'abord expliquer cette présence par les droits ducaux sur le prieuré de Sainte-Croix, car depuis 1096, les ducs d'Aquitaine exerçaient une protection sur Sainte-Croix de Bordeaux et sur ses possessions. Le 15 mars 1165 , le pape Alexandre III rappella à Henri II cette obligation pour faire rentrer les moines de Saint-Macaire dans la sujétion de Sainte-Croix qu'ils rejettaient une fois de plus, apparemment sans résultats immédiats ${ }^{39}$. Depuis au moins le milieu du XI $\mathrm{XI}^{e}$ siècle, les moines de Sainte-Croix et leur protecteur partagaient les prérogatives seigneuriales sur la ville avec les vicomtes de Bezeaumes, seigneurs de Benauges ${ }^{* 0}$. Aussi, lorsqu'Henri II se décida à descendre à Saint-Macaire en 1169, ce n'était visiblement pas pour satisfaire Alexandre III qui patientait depuis quatre ans mais parce que selon toute vraisemblance le vicomte de Bezeaumes, Guillaume Amanieu IV ou son successeur Bernard II de Bouville, participaient au soulèvement des barons aquitains ${ }^{41}$.

Qu'en est-il du Brulhois? D’après J. Clémens utilisant une confirmation de Jean Sans Terre, Raoul de Faye aurait reçu de la part d'Henri II la terre du Brulhois, prise au vicomte de Béarn ${ }^{42}$. S'il est vrai que Jean a séjourné à La Plume en août 1200, pour autant le vicomte de Béarn ne semble pas avoir été dépossédé de cette terre agenaise. Du reste, comme Nicholas Vincent l'a montré, c'est l'honneur de Bramley dans le Surrey que Raoul de Faye reçut, non le Brulhois ${ }^{43}$.

L'arrêt du roi à Saint-Macaire touchait cependant au réseau familial des vicomtes de Béarn. En effet, Guila, la scur du vicomte Pierre IV de Béarn, de Gabarret et de Brulhois (1149-vers 1153), avait épousé un vicomte de Bouville ${ }^{44}$ qui ne peut être que Bernard II de Bouville, vicomte de Bezeaumes, seigneur de Benauges et de Saint-Macaire $(1155-1182 / 1195)^{45}$. Il apparaît aussi qu'au milieu du XII ${ }^{e}$ siècle,

* R.H.F., (voir note 2 ) t. XVI p. $346-347$.

* Archizes Historiques de la Gironde (abrégé en A.H.G.),t. I, p. 225, aecelsiam Sancte Crucis devotioni tuae attentius commendantes, magnificentiam tuam rogamus ... quatinus pro reverentia beati Petri ac nostra et obtentu conumdem fratrum qui inibi commorantur diligas, manuteneas protegas et defendas et contra omnium adversantium impugnationem, et contra homines. Sancti Macharii qui prenominatam aeclesiam multipliciter infestan prsumunt. A.H.G., t. I, p. 227, - monachi at burgenses eis subjecti. :

${ }^{\circ} \mathrm{F}$. Boutoulle, - Le monastère de Saint-Macaire et la politique du duc d'Aquitaine à la fin du XI" siècle *, Actes du sixième colloque l'Entredeux-Mers et son identité tenu à Saint-Macaire, les 27 et 28 septembre 1997 , C.L.E.M., 1998, p. 15-21.

"F. Boutoulle, Socièté laïque en Bondelais et Bazadais des annérs 1070 à 1225 (Pouvoirs et groupes sociaux), Thèse de doctorat de l'université Michel de Montaigne-Bordeaux III s. d., J. B. Marquette, 2001, t. II, p. $951-952$ et t. III, p. 1084.

"J. Clemens, - La maison de Béarn et les Plantagenèts dans le diocèse d'Agen durant la seconde moitié du XII siècle =, Tenes at hommes du sud. Hommage à Piene Tucoo-Chala, 1992, p. 202.

is N.Vincent, " King Henry II and the Poitevins -, dans La leur Plantagenta (1154-1204), Actes du colloque temu à Thouars du 30 avnil au 2 mai 1999, civilisation médiévale VII, Poitiers, 2000, p. 123. En 1165, Gaston V est encore seigneur du Brulhois: il donne avec sa tante la terre de Percemil en faveur de l'hópital de Saint-Jean du Nom de Dieu, près de Nérac. J.Jaurgain, (voir note 16) p. 552, J. Clemens, (voir note 42) p. 204.

"J. Jaurgain, (voir note 16) p. 550. 
la monnaie morlanne, frappée par le vicomte de Béarn, était utilisée en Bezeaumes $^{46}$.

Par la suite, à partir de 1170 , Henri II intervint de moins en moins sur la scène gasconne. En 1170, le prieur de La Réole lui adressa une plainte concernant son fief de Cazes ${ }^{47}$ et peut-être une autre, contre Sanche du Mirail, un bourgeois de La Réole $^{48}$. En 1173, Henri II fit élire un nouvel archevêque de Bordeaux, Guillaume, abbé de Reading dans le Berckshire ${ }^{49}$. Pendant la révolte de 1173-1174, Henri II se faisait appeler « comte de Gascogne * à un moment où il descendit peut-être une nouvelle fois à Saint-Macaire ${ }^{50}$; le roi cherchait à contrer Richard qui venait de prendre la tête du soulèvement des Aquitains. Mais avec le temps, ce dernier s'affirmait de plus en plus dans le duché: après 1174, on ne relève plus d'actions d'Henri II en Gascogne.

\section{Richard, duc d'Aquitaine et les confins de la Gascogne (1170-1199)}

Pendant la trentaine d'années qui séparent 1170 de 1199 , Richard eut la responsabilité de la Gascogne et de l'Aquitaine sauf pendant de brefs intermèdes, d'abord entre 1184 et 1187 avec Aliénor, puis entre 1196 et 1198, lorsqu'il confia le duché d'Aquitaine à son neveu Othon de Brunswick. Jusqu'en 1189, Richard s'occupa activement de la Gascogne: il dirigea prioritairement son action vers les confins méridionaux et orientaux.

Sur le bas Adour

\section{Un intérêt précoce vis-à-vis de Dax et Bayonne}

Les premiers actes de Richard, duc d'Aquitaine, concernent le sud de la Gascogne. A propos de Bayonne, il en a laissé quatre. Les deux premiers, relatifs à la cathédrale, sont conservés dans le Liber Aureus de Bayonne. Il s'agit d'abord d'une confirmation des donations de ses prédécesseurs ducs d'Aquitaine, passée à Poitiers $^{51}$. Le lendemain de l'Epiphanie d'une année non déterminée, mais à l'occa-

\footnotetext{
"Après lui, le vicomte de Bezeaumes et seigneur de Benauges est Pierre de Gabarret, également coseigneur de Langon, en vertu des droits des anciens vicomtes de Gabarret, F. Boutoulle, (voir note 41) t II, p. 951-952 et t. III, p. 1084.

* Cart. La Róole, (voir note 9 ) n'135.

${ }^{n}$ A.H.G. (voir note 39 ), t. 1, p. 188, (1170).

${ }^{4}$ A. Richard, (voir note 28) p. 136; cart. La Rh́ole, (voir note 9) n"125. L'acte n'est pas daté en sorte que rien ne permet d'affirmer qu'Henri ait été saisi de cette plainte en 1162 comme A. Richard le prétend.
Gérard Folium est prieur de La Réole entre 1163 et 1170.

A. Richard, (voir note 28$)$ p. 166.

${ }^{5}$ Cart. Ste-Croix (voir note 9), n*11: - Hec transactio facta in anno ab incarnatione Domini MCIXXXV, Ludovico Franconum ngr, Henrico Anglorum rege, Aquitanie duce, Vasconie comite, Geraldo archiepiscopo Auscitano. ", éd. L. Delisle, (voir note 7) n'CCCLVI, donation en faveur des Templiers d'une église de Londres passée à Saint-Macaire in Gasconia super Genundam, et datée d'après l'éditeur de - février 1173? -; la titulature du roi ne fait pas apparaitre le titre comtal de la Gascogne.

${ }^{3}$ Etudes historiques sur la ville de Bayonne, éd. J. Balasque, t. I, Bayonne, 1862, n* X: Live d'or de Bayonne,

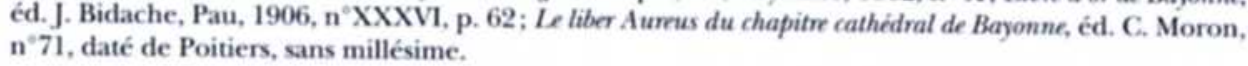


sion de sa première venue à Bayonne (in primo adventu meo), Richard confirma une donation de son prédécesseur Guillaume IX en faveur de l'évêque Raimond de Martres (v. 1122-1125); puis, pour régler un contentieux entre l'évêque Forton Aner (1150-1170) et les bourgeois de Bayonne, Richard donna au prélat la coutume des boucheries de la ville contre l'abandon de ses droits sur la viguerie ${ }^{52}$. En une autre occasion, depuis Bayonne, Richard confirma les privilèges accordés aux cives de Bayonne par son prédécesseur Guillaume IX, du temps de l'évêque Raimond de Martres, et leur imposa de nouvelles obligations (voir infra) ${ }^{53}$. Enfin, dans un acte daté de 1070 (sic), Richard exemptait ses fidelissimi cives Baionensis civitatis du paiement de toute coutume en Poitou, Aquitaine et Gascogne, en raison du service fourni à Henri II et lui-même ${ }^{54}$.

Concernant Dax, le Livre Noir de la ville a conservé deux actes ducaux. Par le premier, passé Bordeaux d'une année non précisée, Richard accordait à ses homines de Dax le droit d'acheter des marchandises sur l'ensemble de sa terre et de les porter in civitate nostra de Aquis". Enfin, par un acte daté de Dax en 1170, Richard donnait à ses fidelissimi cives de la cité de Dax, en raison du service fourni, une franchise de toute coutume aussi bien dans la cité de Dax que sur l'ensemble de sa terre (Poitou, Aquitaine, Gascogne) ${ }^{56}$.

Ces actes posent des problèmes de datation. Cinq d'entre eux présentent des témoins que l'on retrouve d'un texte à l'autre (l'évêque de Dax Guillaume Bertrand, les Aquitains Guillaume Maingot, Foulque de Mastac, le sénéchal du Poitou, Fulcrad d'Archiac, ou le vicomte de Tartas, Raimond Robert ${ }^{57}$, le dernier, passé à Poitiers, ne présente ni témoin ni date. Le millésime 1170, figuré ou suggéré dans deux de ces 6 textes est étonnamment précoce; c'est en mai 1170 (à la Trinité) ou 1172 que Richard fut proclamé duc d'Aquitaine; mais, selon Alfred Richard, les premiers actes datés du jeune duc ne sont pas antérieurs à $1172^{58}$. Aussi, cet auteur

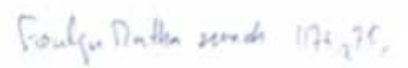

\footnotetext{
${ }^{32} \mathrm{~J}$. Balasque, (voir note 52) $\mathrm{n}^{\circ} \mathrm{XI} ; \mathrm{J}$. Bidache, (voir note 52) $\mathrm{n}^{\circ} \mathrm{XXXVI}, \mathrm{p} .63 ;$ C. Moron, (voir note 52) n'72. Daté de Bayonne, sans millésime.

ss J. Balasque, (voir note 52 ) n"XII ; d'après les Archives de Bayonne AA-1, p. 64.

4J. Balasque, (voir note 52) n"XIII; d'après CompaignE, Chronique de la ville de Bayonne, p. 24.

ss Le Live noir et les établissements de Dax, éd. F. Abbadie, Bordeaux, 1902, p. 173, 351.

s6 Ibidem, p. 179.

${ }^{37} \mathrm{~J}$. Balasque, (voir note 52), $\mathrm{n}^{*} \mathrm{X}$; J. Bidache, (voir note 52) $\mathrm{n}^{*} \mathrm{XXXVI}$; C. Moron, (voir note 52 ) n*71, sans témoin, à Poitiers; J. Balasque, (voir note 52) n'XI; J. Bidache, (voir note 52) n* XXXVI, p. 63; C. Moron, (voir note 52) n'72, à Bayonne un 7 janvier (Guillaume Bertrand, évèque de Dax, Guillaume Maingot, Foulque de Matha, sénéchal du Poitou, le vicomte de Castillon, le vicomte de Tartas, Fulcrad d'Archiac, A. Brun), J. Balasque, (voir note 52) n'XII, à Bayonne (Fort évêque de Bayonne, Guillaume Bertrand évêque de Dax, Guillaume Maingot, Foulques de Matha, sénéchal du Poitou, Raimond Robert vicomte de Tartas, Fulcrad d'Archiac, Guillaume Arnaud de Toxe, Guillaume Aner, A. Brun, Arnaud Decrear clerc) : J. Balasque, (voir note 52) n'XIII, à Bayonne en 1170 (Pierre d'Espelette, évêque de Bayonne, Foulque de Matha, Raimond Robert de Tartas, Arnaud Bertrand de Bayonne, Garsie Bernard de Navailles, Guillaume d'Orthe, Guillaume de Marsan, Loup de Begur, Jean de Saint-Pierre, Jean d'Espelette, Alfonse d'Urtubie, Chicon de Belsunço, Garsie d'Armenditz, Antoine Raimond de Sault. Michel de Parambura, Michel de Saint-Martin, Jean de Garo) ; F. Abbadie, (voir note 56) p. 179, à Dax, en 1170 (Guillaume Bertrand évêque de Dax, Foulques de Matha, Guillaume Maingot, Fulcrad d'Ardriac, Raimond Robert vicomte de Tartas, Pierre vicomte de Castillon, Lob. G. vicomte d'Orthe, Garsie Arnaud de Navailles, Arnaud Guillaume de Marsan): АвварIr. (Fr.), éd. op. cit., p. 173, à Bordeaux (Guillaume Maingot).

s. Aichard, (voir note 28) p. 161-162; J. Gillingham, Richand Carur de Lion, Paris, 1996, p. 93; B. Barrière, -L'anneau de Sainte-Valérie, mythe ou réalité-, dans Valérie at Thomas Bécket. De l'influence des princes Plantagenét dans l'aruvre de Limogrs, Limoges, 1999, p. 11.
} 
proposait de dater les actes intéressant Bayonne et Dax de l'année 1172; J. Balasque optait pour 1174, Fr. Abbadie pour janvier 1178, aussitôt après les sièges de Dax et Bayonne.

Pourtant, on ne peut retenir l'année 1178: la charte passée à Bayonne in primo adventu meo ( $\mathrm{n}^{\circ} \mathrm{XI}$ de l'édition Balasque) a été faite pour l'âme d'Henri, d'Aliénor et des frères de Richard, une volonté qui ne peut pas avoir été prise après 1173 , pendant la captivité d'Aliénor. Du reste, Foulque de Matha était sénéchal de Poitou jusqu'en 1174, après quoi Guillaume Maingot prit sa succession ${ }^{59}$. On ne peut pas non plus retenir le mois de janvier 1171, car Richard célèbrait Noēl 1170 à Bures, près de Bayeux, avec ses parents et ses frères ${ }^{60}$, ni janvier 1173 (il a passé Noël 1172 à Chinon), et puisqu'Aliénor fut capturée en novembre 1173, on peut également écarter le mois de janvier 1174. L'apparition récurrente de certains témoins dans les actes de ce petit dossier nous amène à penser qu'ils ont été émis pendant un intervalle de temps assez bref, entre 1170 et 1172, mais suffisamment long pour qu'apparaisse d'abord auprès de Richard l'évêque de Bayonne Forton Aner, puis son successeur Pierre d'Espelette.

Par cette série de dispositions, Richard réaffirmait une autorité ducale en retrait en Labourd ${ }^{61}$. Entre 1122 et 1126, le duc Guillaume IX avait abandonné à l'évêque de Bayonne, Raimond de Martres et à la cathédrale, la moitié de la cité, ainsi que les padouents, les droits sur les eaux et la $\times \operatorname{mer} *{ }^{62}$. Cependant, le duc ne s'était pas complètement retiré de la ville: une des chartes de Richard évoque les coutumes et les droits concédés jadis par Guillaume IX aux Bayonnais et, nous l'avons vu, le jeune duc pouvait encore donner à l'évêque la coutume sur les boucheries de la villa de Bayonne ${ }^{63}$. Il lui fallait cependant compter avec le vicomte de Labourd dont le prédécesseur avait cédé à la cathédrale, entre 1106 et 1118, l'autre moitié de la cité et des droits sur le port ${ }^{64}$. Au milieu du $\mathrm{XII}^{e}$ siècle, ce vicomte dominait encore un territoire comprenant le Labourd, Arberoue, Ossès, Cize, Irissary, Ilhody, Armendaritz jusqu'aux environs immédiats de Bayonne ${ }^{65}$.

Au terme de ses premières mesures, Richard apparaissait comme le seigneur des Bayonnais, qu'il nommait burgenses, cives meis ou fidelissimi cives Baionensis civitatis. Le service que ces derniers lui avaient apporté est vague; il s'agissait probablement d'une aide contre les seigneurs ayant participé au soulèvement de 1169-1170. A Bayonne, Richard avait toute la viguerie a en sa main *. Par les coutumes qu'il reconnaissait ou qu'il accordait à ses cives, il attendait une participation à l'expeditio derrière le sénéchal, le paiement d'un marc d'argent annuel en remplacement d'une imposition sur les baleines (exactio balenae), le versement d'une

\footnotetext{
50 Ibidem, p. 184

${ }^{6}$ Ibidem, p. 157 .

${ }^{61}$ P. Hourmat, Histoine de Bayonne, Bayonne, 1987; J. Pontet, Histoine de Bayonne, Toulouse, $1991 ; \mathrm{R}$. Mussot-Goulard, - Vicomtes de Labourd, vicomtes de Bayonne -, Rngands sur l'histoin de Bayonne at du sud-ourst aquitain. Mellanges offerts à Piere Hourmat, s. d. J. Pontet, Bayonne, 2001, p. 39-53.

${ }^{6} \mathrm{~J}$. Bidache, (voir note 52) n'XIII; C. Moron, (voir note 52) n'20.

${ }^{63} \mathrm{~J}$. Bidache, (voir note 52) n'XXXVI, p. 62; C. Moron, (voir note 52) n" 71

${ }^{64} \mathrm{~J}$. Bidache, (voir note 52) n'XV; C. Moron, (voir note 52) n"37, 38.

6 J. de Jaurgain (voir note 16) p. 233-247; C. Moron, (voir note 52) n*3, 6, 12, 18, 19, 37, 38, 44, 46, 47. $52,53,54,69,73,74$.
} 
taxe de 2 sous par bateau amarré dans la ville, etc. Assez singulièrement, ce retour marqué de la puissance ducale faisait peu de cas du vicomte de Labourd: Arnaud Bertrand n'apparaît que comme simple témoin dans la franchise accordée aux Bayonnais, pour le reste des actes de Richard il est absent.

Il en allait de même à Dax, où la situation initiale paraît moins défavorable au jeune duc. En écho à l'affirmation de Richard, considérant Dax comme civitas nostra, une vingtaine d'années plus tôt l'archevêque de Bordeaux Geoffroy du Loroux rapportait au régent Suger que la cité était “ propriété du roi * Louis VII, premier époux d'Aliénor (1149). De fait, à la fin du XI $\mathrm{XI}^{\mathrm{e}}$ siècle, les donations du duc d'Aquitaine Guillaume VIII contenues dans le Liber rubeus de la cathédrale portaient sur la cité ou ses environs immédiats, ce que les vicomtes de Dax ne semblent pas avoir été en mesure de faire. Notons qu'en 1170-1172 Richard n'a pas associé aux dispositions prises en faveur des Dacquois ou des Bayonnais le vicomte de Dax, Pierre II, aussi seigneur de Mixe et d'Ostabaret, alors que l'évêque de Dax, Guillaume Bertrand, par ailleurs frère du vicomte de Labourd Arnaud Bertrand, assistait à quatre des actes de la série. On ne peut invoquer ni minorité, ni incapacité de sa part, puisque depuis 1167 sa présence est relevée sur des actes concernant la cathédrale de $\operatorname{Dax}^{66}$. A l'instar d'Arnaud Bertrand de Labourd, Pierre II de Dax a donc été tenu à l'écart des premières mesures ducales.

\section{Les descentes ducales de $1176-1177$ et 1178}

Entre Noël 1176 et janvier 1177, Richard revint vers ces confins avec son ost, dans le cadre d'une expédition dont Benoît de Peterborough et Roger de Hoveden ont rapporté les étapes. Cette action paraît dirigée contre une coalition formée de Pierre II, vicomte de Dax, et de Centulle III, comte de Bigorre et vicomte de Marsan, à laquelle s'est apparemment adjoint Arnaud Bertrand, le vicomte de Bayonne, ainsi que le vicomte d'Orthe.

Parti de Bordeaux, où Richard avait passé Noēl, l'ost ducal s'arrêta d'abord devant Dax, où s'étaient retranchés Pierre de Dax et Centulle de Bigorre ${ }^{67}$. En dix jours, Richard emporta la ville ${ }^{68}$. Le vicomte de Dax trouva la mort dans les combats ${ }^{69}$. Poursuivant sa route, le duc se présenta devant Bayonne, que le vicomte Arnaud Bertrand venait de "fortifier contre lui "; après un nouveau siège de dix jours, la ville finit par tomber à son tour. Dès lors, le vicomte Arnaud Bertrand disparut de la circulation; la vicomté échut à son neveu, Guillaume Raimond de Sault, qui actait encore en 1193 et après qui les vicomtes de Labourd s'éteignirent.

\footnotetext{
6. J. de Jaurgain, (voir note 16), p. 58.

67 Sur Loup Garsie d'Orthe, d'après J. de Jaurgain, ibidem, p. 53 (qui suit la chronique de Dax de Dompnier de Sauviac).

${ }^{6}$ R.H.F., (voir note 2) L. XIII, p. 167: - Et Ricardus comes Pictavie filius gius tenuit curiam suam in Aquitaniam apud Burdegalensem civitatem (...). Comes autem Pictavia, statim post Natale, obsedit Akensem civitatem, quam Petrus vicecomes Aquensis, at comes Bigorniae contra eum munierant, et infra $X$ dies cepit. Et inde promovens exercitum suum obsedit Baionam civitatem, quam Emaldus Bertramnus vicecomes Baioniea contra eum munierat, $t$ infra $X$ dies cepit. -

${ }^{\circ}$ Loup Garsie d'Orthe, en signe de soumission, aurait demandé à Richard d'être le parrain de son fils, Richard dit d'Aspremont:J. de Jaurgain, (voir note 16) p. 53 (d'après la chronique de Dax de Dompnier de Sauviac).
} 
Les motivations de la ligue entre les vicomtes de Dax, de Labour et le comte de Bigorre n'ont pas été réellement déterminées. Remarquons tout d'abord que les villes prises par les barons sont celles où s'était récemment affirmé le retour de la puissance ducale. Il s'agit donc donc prioritairement d'une réaction contre celle-ci. La présence du comte Centulle III de Bigorre à Dax, en 1177, a peut-être été motivée par une parenté avec le vicomte (la mère de Pierre II, Stéphanie, fut mariée une première fois à Centulle II de Bigorre, le grand père de Centulle III) ${ }^{70}$; à tout le moins possédait-t-il le château de Clermont, une forteresse construite par le vicomte Pierre II, au sud-est de Dax ${ }^{71}$.

Après la prise de Bayonne, Richard poursuivit sa route vers l'est et la Cize à travers le Labourd, dont il dut parachever la soumission. Le 9 janvier, il installait son ost devant le castellum de Saint-Pierre qui se rendit le lendemain (le dimanche 10 janvier $)^{72}$. Richard en profita pour faire supprimer les mauvaises coutumes levées sur les pèlerins de Saint-Jacques à Sorges (Sordes?) et Lespurun (Ispoure?). Aussi, en remontant vers la vallée de Cize jusqu'aux Portes de l'Espagne, Richard se montrait en Mixe et en Ostabarret, deux seigneuries pyrénéennes relevant des vicomtes de Dax, mais également sur la route de Sorde à Saint-Jean-Pied-de-Port, un axe dont nous avons vu l'importance stratégique.

Ce faisant, Richard agissait aussi sur un autre registre. Ainsi qu'en témoigne le Guide du pèlerin que Richard connaissait certainement, après Sorde, où convergaient les voies de Tours, Vezelay et du Puy, l'itinéraire du jacquet devenait plus éprouvant: les sinistres passeurs de Sorde, les extorsions des seigneurs péagers d'Ostabat ou de Saint-Jean-Pied-de-Port, les huit milles d'ascension vers Roncevaux représentaient autant de douloureuses stations avant la Croix de Charlemagne et le réconfort de l'hospice de Roncevaux. Aussi, en conduisant cette démonstration de force et en faisant rendre gorge aux seigneurs qui rançonnaient les pèlerins, Richard donnait l'impression de placer sous sa protection un des tronçons les plus prestigieux de l'itinéraire vers Saint-Jacques et de suivre la geste de Charlemagne. C'est d'ailleurs avec un souci affirmé de la propagande politique que le 2 février, à peine rentré à Poitiers, Richard adressa un messager à son père pour lui faire part de ses succès ${ }^{73}$.

Pourtant Richard n'en avait pas terminé avec ces confins. En 1178, il dut revenir à Dax, à la tête d' " une grande armée ", parce que le comte de Bigorre venait d'attaquer la ville. Les citoyens de Dax, dans des circonstances que l'on ignore, parvinrent cependant à capturer l'asssaillant et à l'emprisonner (cives ejusdem civitatis). Sur l'intervention du roi d'Aragon, Alphonse II, qui se porta caution pour la libé-

\footnotetext{
J. de Jaurgain, (voir note 16) p. 382 et 552.

$"$ Cartulaire de la cathédrale de Dax, Liber Rubeus, (XF-XIt siècles), éd. G. Pon, J. Cabanot, Dax, 2004. n"12.

${ }^{72}$ R.H.F., (voir note 2) t. XIII, p. 167 (Benoit de Peterborough), - Et inde promovens romcitum suum usque Cizan, que nunc Portac Hispanie vocantur, obsedit castellum Sancti Petri ct eadem die copit, scilicet dominica proxima post Epiphaniam, et penitus destruxit omnes malas consuctudines que inducte crant apud Songes at apud Lespurun, ubi mos arat depraedare pergnino sancto Jacobi. Et sic pacificatius omnibus provinciis, in purificatione Sancte Marie venit Pictavim.... . . Le castellum de Saint-Pierre est celui de Saint-Jean le Vieux, M. Goyhenetche, Histoire générale du Pays Basque, t. I, Bayonne, 1998, p. 279.

is R.H.F, (voir note 2) t. XIII, p. 167, - Et sic pacificatis omnibus provinciis, in Punificatione sancte Marie venir Pictavim a inde dirxit nuncium suum in Angliam ad Regem patrem suum. = C'est certainement sur la base de ce rapport qu'a été bâti le récit de Benoit de Peterbourough. Sur la popularité du culte de Saint-jacques de Compostelle chez les Plantagenêts, J. Gillingham, Richard Carur de Lion, Paris, 1996, p. 80-81.
} 
ration de son amicus, Centulle fut relaxé contre l'abandon de Clermont et du castellum de Montbrun ${ }^{74}$.

Après cette mémorable expédition, les interventions de Richard au sud de la Gascogne s'espacèrent nettement. En juillet 1179, faute de subsides, le duc dut licencier ses mercenaires brabaçons, basques et navarrais, qui, en regagnant leurs foyers, pillèrent les faubourgs de Bordeaux ${ }^{75}$. En 1181, Richard était à Saint-Sever où il accepta le pardon de Vivien de Lomagne. Pendant son règne (1189-1199), il fit adopter un établissement pour les Bayonnais (la charte dite des malfaiteurs) sur la répression des crimes et délits commis dans la ville et sur le territoire de la vicomté (voir infra) ${ }^{76}$. De même, nous ne pouvons pas dater la construction par le roi Richard du castrum d'Uza, au sud du pays de Born, sur la directe ducale * in sua propria terra ", ni les coutumes accordées à ses habitants évoquées par une lettre des prud'hommes d'Uza en $1249 .{ }^{77}$

\section{Affirmation de l'autorité ducale sur la vallée de la Garonne}

Dans les confins orientaux de la Gascogne, marqués par la permanence de la "guerre de Quarante ans " contre le conte de Toulouse (1156-1196), la vallée de la Garonne représentait un axe vital. Dans un premier temps, les efforts de Richard se sont portés vers l'Agenais et la Lomagne.

\section{Richard et Agen}

Le site de Castillon ou Castillou-sur-Agen, sur une éminence calcaire au dessus de la ville (fig. 1) ${ }^{78}$, fut l'objet d'un siège mémorable rapporté par Robert du Mont et Benoît de Peterborough. Ayant reçu l'ordre de rabaisser les châteaux qui s'étaient élevés contre son père, Richard se porta contre celui de Castillou, fortifié par

7 R.H.F., (voir note 2) t. XIII (Benoit de Peterborough), p. 178. - Eodem anno, Ricandus Dux Normanniae a Aquitanie, post transfretationem Henrici mgis Anglie patris sui, cum magno arrcitu in Pictaviam profectus, ad Assiensem civitatem venit : a imento ibi Comite Bigorviae incarcento, quem cives giusdem civitatis incarreraverant, magno fluctuavit gaudio. Sed Amfussus Rex Arragonum, dolens quod amicus suus Comes Bigorniae tenebatur in vinculis, venit ad prefatum Ducem; $t$ sollicitus ut amicus suus a canere prarfati Ducis libcrartur, fidgussor pro illo exstitit adversus Ducem, quod ille voluntatem Ducis at patris ipius Rrgis Anglie facert Et insuper praedictus Comes Bigorniae pro liberatione sua tradidit praenominato duci Clarum-montem et castellum de Munbrun. *

${ }^{75}$ R.H.F, (voir note 2) t. XIII, p. 322. - Quidam Basdi at Navarnenses at Brbenzones venerunt ad urbem Bunde galensem a ipsam urbem vastaverunt in suburbiis flamnis at rapina. = C'est à cet épisode qu'il faut certainement rattacher la destruction des faubourgs de Bordeaux rapportée par la chronique de De Lurbe (placée en 1179) et que Yíes Renouard lie à l'attaque du roi de Castille, en 1205-1206. Y. Renouard, s. d., Histoine de Bordeaux médicual, Bordeaux, 1963, t. 2, p. 26.

* J. Balasque, (voir note 52) n" XIV. Acte passé en présence de Guillaume Bertrand évêque de Dax (1168-1203) et Bernard de Lescar évêque de Bayonne (1186-1213). Peut-ètré édicté lors du dernier séjour de Richard à Bayonne avant de partir pour la croisade (voir infra).

" Royal and other historical letters illustrative of the reign of Henri III, éd. W. W. Shirley, t. I, (1216-1235), London, 1862, n* CCCCI.XII, p. 57-58, - Serenissimo ac magnifico vimo domino Henrico, Dri gratia ngi Anglie, probi homines Castri Dusar, salutem (...) Noverit vestra majestas ngis quod dominus nx Richandus fecit castrum Dusar, in sua propria terra et populavit idem suis propriis hominibus bonis moribus a consurtudinibus, et tenuit cum quantum vixit at dimisit eum domino rgi Johanni, sicut aliam terram suam (...). .

จ Il est marqué par une butte calcaire dominant la vallée de $400 \mathrm{~m}$ de long coupée par un fossé et d'une largeur variant de 20 à $80 \mathrm{~m}$. En 1482 Jean de Beauville rend hommage à l'évêque d'Agen pour le * castrum de Castelhono prope Agrnni=, f. J. Clemens, (voir note 42) p. 206. 
Arnaud de Bouville. Il s'y dirigea vers la fête de saint Jean Baptiste, avec une grande armée et des engins de siège; au bout de 2 mois le castrum tomba et Richard put capturer 30 milites $^{29}$. D'après une tradition rapportée par une enquête de 1311 , le duc aurait fait raser le château et semer du sel pour empêcher toute reconstruction. Ces traditions locales, repérées également dans des actes de la région ${ }^{80}$, ont permis à J. Clémens de montrer que le récit de Robert du Mont est erroné sur trois points: le responsable du siège est Richard et non Henri; il a duré deux mois et non une semaine; il se situe en 1175 et non en 1161. Comme la prise de Saint-Macaire, six ans plus tôt, la prise de Castillou semble dirigée contre les Bouville, alliés, nous l'avons vu, aux vicomtes de Béarn ${ }^{81}$. Peu après ce fait d'armes, en présence d'Henri de Penne, constabularius Castillionis, Richard, se présentant comme filius Henrici rgis Anglie et comte de Poitiers, prit sous sa protection le prieuré de Sainte-Livrade, situé sur le Lot, dépendant de l'abbaye de la Chaise-Dieu ${ }^{82}$.

Placée aux avant-postes du conflit contre Toulouse, Agen a par la suite suscité beaucoup d'intérêt chez Richard. A l'exemple de ses prédécesseurs ducs d'Aquitaine, il confirma aux évêques la * comitalie * et la monnaie, c'est-à-dire l'exercice des prérogatives publiques qui leur avaient été abandonnées depuis le $\mathrm{XI}^{\mathrm{e}}$ siècle $^{83}$. Mais le trafic sur la Garonne relevait encore du duc ou avait été récupéré: ce fut pour Richard un vecteur déterminant. A l'occasion d'un arrêt non daté à Agen, le comte de Poitiers prit sous sa protection l'abbaye de Grandselve, située en Toulousain, ainsi que ses biens ${ }^{84}$. Cet établissement particulièrement choyé a également reçu du duc la liberté pour une embarcation de descendre et remonter la Garonne, une fois par an, sans acquitter de coutume, sur les ports relevant de Richard; le duc put en informer son sénéchal d'Agenais (senescallus Aginnensi) et ses «chers et fidèles citoyens d'Agen ${ }^{85}$. Ces derniers étaient donc associés à la levée des coutumes

\footnotetext{
R.H.F., (voir note 2) t. XIII (Benoit de Peterborough) p. 163: - Eodem anno, Ricandus comes Pictavie, filius Henrici mgis Anglie, castella Pictaviae, quae contra patrem suum tempore hostilitatis infortiata vel ritenta fuerant, per praeceptum patris sui in nihilum redegit et circa festum sancti johannis Baptiste, congrgato magne excritu, obsedit Castellionum supra Agiens, quod Armaldum de Bovilla contra cum munierat, nec ai neddere voluit. Et paratis ibi machini sui bellicis, infra duos menses copit illud, et triginta milites in eo copit et in manu sua ntimuit. R.H.F., (voir note 2) t. XIII, p. 306 (Robert du Mont - 1161 -). - Inde, datis induciis post festum S. Johannis, Henricus rex Anglorum pernxit in Aquitaniam, at inter alia que strenue gessit, Castellionem super urbem Agennum, castrum scilicet natura et artificio munitum, obsedit; et infra unam septimanam, in festivitate $S$. Laurentii, admi. rantibus et perterritis Wasconibus, cepit. :

"Donation en faveur du Temple d'Argentens, - tempore Pictaviensi consul insedit Castillon, Elia de Castillon manente episcopo Agrnnensi=, J. Clemens, (voir note 42) p. 207.

${ }^{n}$ Vers 1170, Richard aurait également confirmé un arbitrage rendus par Hélie de Castillon et Garsie de Benquet, respectivement évêques d'Agen et de Bazas, condamnant les habitants de Condom contre l'abbé de Condom: J. Clemens, (voir note 42) p. 207, d'après J. Gardere, Histoire de la seigneurie de Condom, Condom, 1932, p. 21.

${ }^{2}$ BN lat. 12751, p. 583-584, B.N lat. 12678, f. 232 v. On relève parmi les témoins, Guillaume Maingot, Foulque de Mastac et Foulque d'Archiac. Nous remercions vivement Nicholas Vincent, qui prépare une édition des actes de Richard, pour nous avoir communiqué sa transcription.

${ }^{s}$ A. Pottast, Regesta pontificum romanorum (1198-1304), vol. 1, 1874, Berlin, p. 219, n'2554 (confirmation par Innocent III, le 30 juin 1205). P. Wolf, - Evêques et comté d'Agen au XI' siècle -, Villeneutesur-Lot et l'Agenais, Actes des XTV a XVIt congrès d'ítudes nigionales tenus à Villenerue-sur-Lot les 13, 14 at 15 mai 1961 . Agen, 1962, p. 115-120.

${ }^{*}$ B.N. Doat, 80 f. 31 r-v.. Communication N. Vincent.

${ }^{55}$ BN Lat. 11010, f. 42, v., et 145 v.; B.N. Doat, 80, f. 305v., sans date. Communication N. Vincent.
} 
à l'exemple, semble-t-il, de la firma burgi anglo-normande, un système qui permettait aux villes de recueillir elles-mêmes les impôts dus au roi ${ }^{86}$.

Le 12 novembre 1189, depuis Londres, Richard adressa une lettre patente à l'évêque d'Agen, à ses ministri et à ses fidèles de la ville, les informant qu'il ne prendrait sur le nouveau pont d'Agen, nul pontagium, passagium, exactiones ou consuetudines; en outre, il accordait au «commun conseil de la ville * (communi consilio ville Agenni) la libertas d'élire deux pontenarii chargés de recueillir les aumônes devant financer le chantier ${ }^{57}$.

\section{En Lomagne}

En 1181, d'après Geoffroy du Vigeois, Richard à la tête de l'ost des Gascons mit le siège devant Lectoure où s'était enfermé le vicomte de Lomagne, Vivien II; celuici obtint finalement le pardon ducal et Richard le fit chevalier, le 15 août de la même année à Saint-Sever ${ }^{48}$. Les raisons de cette nouvelle descente ducale ne sont pas claires. Elles sont certainement liées à la guerre contre le comte de Toulouse, puisque les vicomtes de Lomagne étaient également vicomtes d'Auvillar. Mais là encore Richard agissait contre les réseaux des vicomtes de Béarn, puisque les vicomtes de Lomagne, voisins du Brulhois, étaient parents des anciens vicomtes de Gabardan depuis le $\mathrm{XI}^{e}$ siècle $^{89}$.

En cette occasion, Richard aurait apporté un concours militaire au comte d'Armagnac et de Fezensac, Bernard IV, dans une entreprise dirigée contre l'archevêque d'Auch, Géraud de La Barthe. La notice qui relate ces événements a été rédigée un siècle après les faits et présente quelques erreurs que Jaurgain a corrigées ${ }^{90}$. D'après ce texte, Bernard IV et son fils Géraud, ont eu recours à une armée réunie par «le comte de Poitiers, R. " (ce ne peut être que Richard), alors stationnée à Lectoure, pour attaquer l'église Sainte-Marie d'Auch, l'archevêque et les chanoines. Fort de ce concours, Bernard et son fils purent s'emparer de la cathédrale, incendier le cloitre, ainsi que les domus des chanoines et de l'archevêque ${ }^{91}$.

Apparemment Richard n'a pas participé en personne à cette offensive contre l'archevêque; il a laissé ses hommes, des mercenaires certainement, suivre le comte d'Armagnac, seigneur pour partie de la ville d'Auch. Il renouait avec un comte

\footnotetext{
3. Boussard, Le gouvernement d'Henri II Plantagenét, Abbeville, 1956, p. 167-177 et 187: dans les années 1176-1180, d'après les rồles de l'Echiquier, un grand nombre de villes normandes ont affermé les revenus royaux.

${ }^{87}$ Chartes. Premièr śrie (1189.1328), Archives municipales d'Agen, éd. A. Magen, G. Tholin, Villeneuvesur-Lot, 1876, $\mathrm{n}^{\circ} \mathrm{I}$.

s.H.F., (voir note 2) t. XII, p. 449 (Geoffroy du Vigeois), - Richandus interim cum hoste Vasconiam petens Ledoram occupavit, quoadusque Vivianus vicecomes de Lomania portam clausam seseque tradens, ab eodem cingulum percipert apud S. Severum instante Virginis Mariae Assumpione= ; J. Gillingham, (voir note 74) p. 124. "Les vicomtes de Lomagne, alliés au Gabarret, avaient abandonné leurs droits en Gabardan et en Brulhois: J. de Jaurgain, (voir note 16) p. 19-23.

so Ibidem, p. 154.

"1 R.H.F., (voir note 2) t. XII, p. 390; Gallia christiana t. I, inst. p. 164. - Postmodum vero, idem Bernandus in solita malitia et pertinacia persenerans, in nullo factae stetit compositioni, clericum at scriptorem D. Archicpiscopi copit at inturravit: et resuscitatis inter eos inimicitiis, pro 00 maxime quia ipse Bernardus at Geraldus praescriptam eclesiam dominam at matrem suam sibi subjugare intendebant, jura Archiepiscopalia $t$ Canonicorum ubique invadentes; prefactus Geraldus praescriptam Ecclesiam dominam ausu diabolico, cum exerifu Raimundi (sic) Comitis Pictaviensis qui tunc Lectorae arat, expugnavit et cepit, eamque claustra et domos Canonicorum et Archie piscopi et Canonicis, apposito igne combussit. -
} 
dont le père, Géraud III, fréquentait déjà le duc d'Aquitaine Guillaume X en $1128^{92}$. Richard s'assurait en plus de la bienveillance du plus puissant des seigneurs des confins orientaux de la Gascogne, alors que la guerre contre Toulouse battait son plein. Géraud de La Barthe ne tint pas rigueur à Richard de son engagement en faveur de son adversaire: en 1190 il assuma, d'après Roger de Hoveden et avec l'évêque de Bayonne, le commandement de la flotte royale en partance vers la Terre Sainte ${ }^{93}$.

\section{En Agenais occidental}

Dans la même période, Richard fixa les coutumes de Marmande, en Agenais, sur la rive droite de la Garonne, une ville dont il passe pour être le fondateur. Pourtant, à la fin du XII ${ }^{e}$ siècle, le site de Marmande n'était pas inhabité car il correspond à l'ancien castrum de Granon où un concile avait été réuni en 673-675. Nous n'avons que des témoignages indirects mais concordants de cette "fondation *, étudiée par $\mathrm{Ch}$. Higounet ${ }^{94}$. Elle est rapportée par une requête du prieur de Marmande, datée de 1320, demandant que fussent respectées les dispenses de péage sur le blé et sur le vin accordées à l'abbé de Clairac et à la maison de Saint-Pierre de Granon, par Richard, comte de Poitiers et duc d'Aquitaine, « lorsqu'il établit la ville de Marmande $*$. Un vidimus de 1340 de la charte de franchise de la ville rappelle cette origine ducale et place la fondation en 1182. Cependant, une charte d'Agen, datée de 1222, attribue le péage de Marmande à Henri II.

D'après Charles Higounet, on peut au moins attribuer à Richard les premiers statuts du peuplement qui se développait en ce lieu, la fixation des tarifs du péage et les dispenses accordées au prieuré de Granon, en dédommagement, peut-on penser, de la perte de ses droits sur le péage ou sur le castrum; cependant, les circonstances de la prise de contôle du site restent inconnues (spoliation, conquête...). Si l'on en juge par le rôle que Marmande joua par la suite dans la croisade des Albigeois, cette $\propto$ fondation $*$ fut une réussite.

Sur l'autre rive de la Garonne, à $6 \mathrm{~km}$ en aval de Marmande, mais dans une période que nous ne saurions préciser, Richard s'est ménagé un autre point d'appui à Couthures-sur-Garonne, à la limite du Bazadais et de l'Agenais. Celui-ci est évoqué dans un mandement de Jean sans Terre, daté 22 août 1200, par lequel le roi faisait savoir aux prud'hommes de Couthures et Labarra (non localisé) qu'il venait de donner à Arnaud Gast et ses héritiers la "terre de Couthures et de Labarre * avec leur honor et qu'ils leur fallait répondre devant ledit Arnaud comme ils l'avaient fait, jadis, devant le roi Richard ${ }^{95}$. Le château de Couthures, attesté dans un mandement du 2 octobre 1214, a peut-être été construit par Richard ${ }^{96}$. Faute de données

\footnotetext{
${ }^{27}$ G.C.S.M., n*43. Confirmation de la sauveté de La Sauve-Majeure par le duc Guillaume X, lequel * hor signum confirmationis in karta de salvitate manu sua conscripsit aliudque Geraldo comiti de Armanag facere mandavit. :

${ }^{93}$ Chronica magistri Rogeri de Hovedene, éd. W. Stusas, Londres, 1868, p. 36.

"C. Higounet, - Le développement urbain et le rôle de Marmande au Moyen Age n, Rronue de l'Agenais, 1952, rééd. Paysages et villages neufs du Mogen Agr, Bordeaux, 1975, p. 326-334; J. Clemens, Marmande. Coll. Atlas historique des villes de France, Paris, 1985.

ss Rotuli chartarum in turr Londinensi asservati, vol.I, pars I, anno I199.1226, éd. T. D. Hardy, Londres, 1837, (abrégé en Rot. chart.), p. 74 b.

` Ibidem, p. 201.
} 
précises sur ce secteur du Bazadais nous ne savons pas de qui relevait cette localité antérieurement. Mais, située entre Marmande et La Réole (12 km), Couthures constituait une pièce supplémentaire dans le dispositif de contrôle de la vallée.

\section{En Bazadais (1185-1189)}

Sur l'axe garonnais, Richard ne pouvait pas se désintéresser de La Réole. Siège d'un prieuré de Fleury-sur-Loire dont le vicomte de Bezeaumes avait été avoué, ce bourg prieural était au XII ${ }^{e}$ siècle la ville la plus importante du Bazadais ${ }^{97}$. Richard s'y arrêta entre 1185 et $1189^{98}$. Il pouvait faire valoir un droit d'hébergement, une procuratio tarifée dans les Anciennes coutumes de La Réole rédigées vers 1187 $1188^{99}$. Par ailleurs, comme on l'a déjà vu, la justice ducale s'est intéressée aux affaires du prieuré; en 1170 , le prieur avait adressé deux plaintes à Henri II contre un bourgeois qui refusait d'acquitter les lods et ventes et contre les seigneurs qui récusaient les droits du prieur sur le fief de Cazes. Enfin, à une époque que l'on ne saurait déterminer, le duc Richard franchit une étape capitale en prenant le prieuré et ses biens sous sa défense et sa garde ${ }^{100}$.

Le séjour de 1185-1189 permit à Richard de renforcer sa position dans la ville. D'après une enquête menée en 1236 sur ordre d'Henri III, le prieur de La Réole avait été dépossédé de la justicia sanguinis par Richard parce qu'il n'avait pas pu s'opposer aux menées d'une bande de juvenes qui venaient de voler un bourgeois, Etienne de Lavison, par ailleurs compater de Richard ${ }^{101}$; la victime ayant déposé plainte à la cour de Richard, le comte, de passage à La Réole (ad Regulam accedens), en profita pour s'emparer de la « justice du sang * (justicia sanguinis) et pour installer un prévôt en ce lieu.

Enfin, Richard ou Henri ont implanté une tour à La Réole ${ }^{102}$. Selon Dom Maupel, un moine mauriste qui a rédigé au XVIII' siècle une histoire du prieuré de La Réole en s'appuyant sur des pièces du cartulaire aujourd'hui disparues, Henri II

\footnotetext{
${ }^{n}$ Les portes de ce bourg sont attestées en 1187-1188 dans les Anciennes coutumes de la ville, éd. M. Malherbe, Les institutions municipales de la ville de La Réole, des origines à la ruvolution française, thèse de doctorat, s.d., P. Jaubert, Université de Bordeaux I, 1975, p. 725, art. 35. Il y avait certainement une enceinte, comme à Saint-Sever à la mème époque: P. D. Du Buisson, Historiae monasterii S. Severi libri X, 1681, éd. J. F. Pedegert et A. Lugat, Aire-sur-l'Adour, 1876; J.C. Lassere, Saint-Sever, Atlas des villes de France, 1982; J. B. Marquette, -La révolution de Saint-Sever en 1208 -, Saint-Sarr, millénaire de l'abbaye, 1986, p. 55-75.

" Recueil d'actes nlatifs à l'administration des mis d'Angletere en Guyenne au XIIt siz̀cle. Recognitiones feodonum in Aquitania, éd. C. Bemont, Paris, 1914 (abrégé en Rec. frod.), n"495, et A.H.G., (voir note 39) t. III, p. $18-19$.

"Anciennes coutumes de La Réole, éd. M. Malherbe, (voir note 98) n`38, - item cum comes procurationem accipiet, claviger per totam villam manulevabit porcos at capiet gallinas per domos. -

100 Cart. La Rrole, (voir note 9) n'113, - go suscepi in protectione, custodia, at defensione mea, ecclesiam Sancti Petri de Reola *; acte passé à Guitres.

${ }^{101}$ Dom Maupel, - Sancti Petri de Regula, rgalis prioratus, historico chronologica sinopsis seru de rbus notatu dignis incliti monasterii Regulensis *, Archives Historiques du departement de la Ginonde, t. XXXVI, Bordeaux, 1901, p. 27-29, - predictus Stephanus accessit ad dominum Richardum comitem Pictavensium qui erat compater predicti Stephani de Lavison et valde cum diligebat, quia crebro et libenter pecuniam ci mutuo dabat, apud quem querimoniam deposuit. Qui comes, ad Regulam accedens et prefatos duodecim juvenes non inveniens, quia propter timorem adventus sui aufugerant, posuit in villa de Regula proposituram et spoliavit colesiam justitia sanguinis violenter"; M. Malherbe, (voir note 98 ) p. 172.

${ }^{10}$ La turris est signalée dans les mandements royaux de 1220-1221 (Patent rolls of the rign of Henri III preserved in the P.R.O., A.D. 1216-1225, Londres, 1901, p.275-276).
} 
aurait fait construire un castrum dans les jardins des bénédictins; l'église abbatiale, qui dominait la nouvelle forteresse fut détruite et rebâtie à faible distance ${ }^{103}$. Une pièce du cartulaire de La Réole montre en effet qu'en 1187 le prieur de La Réole dut s'entendre avec le chapelain de la nouvelle église de la Madeleine, après le déplacement de l'église abbatiale ${ }^{104}$.

La place acquise par La Réole dans les affaire ducale apparaît de manière éclatante quelques mois avant le départ pour la troisième croisade. Richard y séjourna du 3 au 4 février 1190 et en cette occasion confirma les privilèges des abbayes de la Sauve-Majeure, de Saint-Sever et de l'ermitage de Verdelais ${ }^{105}$, en présence du baronnage gascon et d'une partie de l'épiscopat (voir infra). Le nouveau roi ne s'étant apparemment pas arrêté à Bordeaux lors de cette dernière descente, La Réole devait avoir acquis à ses yeux le rang de centre politique de la Gascogne ducale.

En Bazadais même, La Réole éclipsait la cité épiscopale dans le dispositif ducal. Nous n'avons conservé qu'un acte du duc Richard, malheureusement non daté, passé à Bazas (apud Vasatum), une prise sous protection ducale de l'abbaye cistercienne de Fontguilhem accompagnée d'une autorisation à acquérir des biens des hommes francs relevant du roi (quicquid acquirere poterunt in francalibus meis jus meum eis dedisse) ${ }^{106}$. Cette donation, qui révèle une partie de la directe ducale en Bazadais, montre aussi que le duc pouvait s'arrêter à Bazas, dans une cité dont l'évêque était seigneur $^{107}$.

Le même doute plane sur les droits de Richard à Captieux où il s'arrêta en 1186 (Capsius) pour confirmer les privilèges de la cathédrale Saint-André de Bordeaux ${ }^{108}$. Située à la limite du Bazadais et du diocèse d'Aire, Captieux représentait une clé sur l'axe Langon-Bazas-Aire à travers la Lande et à la limite du Gabardan. Nous ne savons pas quand cette seigneurie fut intégrée aux possessions des vicomtes de Béarn, ce qui est avéré au milieu du $\mathrm{XIII}^{e}$ siècle $^{109}$. Il reste que l'importance des droits ducaux à Captieux même dans la seconde moitié du XIII ${ }^{e}$ siècle ouvre la possibilité que cette place ait pu être encore, à la fin du $\mathrm{XII}^{\mathrm{e}}$ siècle, intégrée à la directe ducale, avant d'en être distraite ultérieurement ${ }^{110}$.

\footnotetext{
${ }^{103}$ Dom Maupel, (voir note 102) p. 26, - jussit apud Rrgulam extrui castrum loco maxime apto, occupatusque est ab rius ministris hortus monasterii, in quo arx nova edificata est. Sed quia ecclesia divi Prtri altitudine ct firmata sua multum obesse posse videbatur, Angli eam destruxerunt aliamque humilionem at remotionem edificarunt. ${ }^{10}$ Dom Maupel, (voir note 102) p. 26; cart. La Ríole, (voir note 9 ) n"92 (A.H.G., voir note 39) t. II, $\mathrm{n}^{\circ} 105$.

${ }^{10}$ G.C.S.M., n¹106; E. du Laura, (voir note 7) p. 127 ; Rév. P. de Rouvray, Histoire du pílerinage Notre Dame de Vendelais, 1953, p. 281 ; P. D. du Buisson, (voir note 98) p. 200 ; L. Landon, Itinerary of King Richand I, Londres, 1935, p. 25; J. Gillingham, (voir note 74) p. 195.

165 Róles Gascons, éd. C. Bemont, t. 2 (1273-1290), Paris, 1900, n'1567, p. 484-485. Le duc confirme également la donation de la terre de La Val et de Musti et de Lavazan.

${ }^{107}$ J. B. Marquette, - Notes sur I'histoire de la ville de Bazas au XIII" siècle *, Cahiers du Bazadais, n"65. $2^{e}$ trimestre 1984, p. 3-49; n"66, $3^{\circ}$ trimestre 1984, p. 3-42; Idem, - Le site épiscopal de Bazas. Etat de la question -, Cahiers du Bazadais, n"98-99, 34-4" trimestre 1992.

ios Gallia Christiana, t. II, col. 285-286.

109 P. Tucoo-Chala, (voir note 20) p. 151. Promesse d'hommage faite par le vicomte Guillaume II au sénéchal de Gascogne, in plena curia, à Captieux, le 22 février 1222 (a.st.). Le texte ne précise pas cependant les droits du vicomte à Captieux.

${ }^{110}$ Róles Gascons(1254-1255), éd. C. Bemont, supplément au tome I, Paris, Documents inédits de l'histoire de France, 1896, n4389 (1255), approbation de la donation du castrum; Rec. feod, (voir note 99 ) n"235 et 247 (présence d'hommes francs du roi participant à la queste des hommes francs du Bazadais). F. Boutoulle, (voir note 41) p. 169.
} 


\section{En Entre-deux-Mers bordelais}

En Entre-deux-Mers bordelais, Richard disposait d'un vaste domaine direct s'étendant, d'après l'enquête de 1237 , sur près de 40 paroisses ${ }^{111}$. D'après la même enquête, les hommes francs de cette région bénéficiaient de coutumes et de libertés depuis « le temps des rois Henri et Richard ", ce que confirme une lettre du roi Jean, datée du 16 avril 1214 , en faveur des «prud'hommes * de l'Entre-deux-Mers ${ }^{112}$. Parmi ces droits et franchises l'enquête énumère le service militaire des agricolae, limité aux seuls sièges, un concours à l'arrestation des malfaiteurs (au cri d'appel «biahora ), une aubergade annuelle en faveur des agents ducaux, ainsi qu'une quête collective de 40 livres. Cependant, l'enquête n'attribue pas explicitement ces coutumes à Henri ou Richard qui sont seulement présentés, en 1237, comme des référents chronologiques ${ }^{113}$.

Sur les confins orientaux de la directe, Richard semble avoir réactivé la vieille tour de Bisqueytan, située dans la paroisse de Saint-Quentin-de-Baron. Cette tour, datée selon des récentes fouilles du début du $\mathrm{XI}^{e}$ siècle, est citée pour la première fois dans un acte du cartulaire de La Sauve-Majeure des années 1155-1182, alors qu'elle relevait, pour partie, du comte de Poitiers ${ }^{114}$.

En faveur de l'abbaye de La Sauve, Richard renouvela la protection accordée par son père en 1156, d'abord à une date indéterminée depuis Niort, puis le 3 février 1190 à La Réole ${ }^{115}$. Le 4 février 1190, le sénéchal de Gascogne Hélie de Celles recevait l'ordre de faire respecter ces dispositions ${ }^{116}$. A l'occasion de la canonisation de Gérard de Corbie, fondateur de La Sauve-Majeure, le 27 avril 1197, Othon de Brunswick, neveu de Richard et éphémère duc d'Aquitaine, reçut du pape Célestin III une invitation à faire promulguer par l'épiscopat et en synode la fête de saint Gérard ${ }^{117}$. Othon adressa donc une première lettre aux archevêques et évêques de son ressort (ducatum Aquitanie) pour le leur demander et les informer qu'il avait institué une foire le jour de l'Elevation du saint, le 21 juin. Puis le 9 mars 1198 (n.st.), depuis Bazas, Othon enjoignit à ses sénéchaux, prévôt et baillis de faire

\footnotetext{
II Petit cartulaine de La Sauù-Majeur, Bibliothèque municipale de Bordeaux, ms 770 (abrégé en P.C.S.M.). p. 126-131; Rec. feod., (voir note 99) n'531, 537, 541, 542-543, 588, 589, 592, 601, 604, 612, 631, 646, 647 . 665,$669 ;$ F. Boutoulle (voir note 41) t. I., p. 167.

11 Rotuli litterarum patentium in turri Londinensi asservati, éd. T. D. Hardy, Londres, 1835, vol. I, pars I. 1201-1226, p. 112 b.

"11 Le méme type de prestation est demandé aux francs du Bazadais, mais elle n’apparaît pas dans la documentation avant les Recognitiones de 1274.

"14 G.C.S.M. n'527, - terram quam habebat juxta molendinum d'Avaron ex parte turnis de Biscaita quam tenebat prefata mulier de comite Pictavensium. - Pierre Savaric et le prévôt de La Sauve, Raimond de Carignan, sont cités dans d'autres actes du cartulaire des années $1155-1182$; F. Boutoulle, J. L. Piat, - La tour et le château de Bisqueytan en Bordelais: une forteresse ducale révélée par l'archéologie et par les textes (XI*-XIV siècles) -, Résidences du pouvoir et pouvoirs de la nésidence, Actes du colloque de Pau des 3, 4 et 5 octobre 2002, Aquitania, à paraiture.

${ }^{115}$ BN ms lat. 12751, non daté, communiqué par N.Vincent. E. du Laura, (voír note 7) p. 127.

${ }^{116}$ E. du Laura, (voir note 7) p. 126-127. Le 17 mars 1190, depuis Chinon, Richard rappela à Hélie de Celles ses obligations, en reconnaissance des prières faites par les moines à son intention (E. du Laura, voir note 7) p. 126-127).

${ }^{117}$ Patrologiae cursus completus, éd. J.P. Migne, t. CXLVII, Paris, 1853, col. 1019-1020; E. du Laura, (voir note 7) p. 286.
} 
respecter la fête de saint Gérard, la foire et la securitas de ceux qui se rendraient à La Sauve en cette occasion ${ }^{118}$.

Vis-à-vis de Saint-Macaire et Benauges, la politique de Richard restait dans la ligne fixée par Henri II. Le 4 février 1190, depuis La Réole, il confirma les donations passées par les vicomtes de Bezeaumes, Guillaume Amanieu et Bernard de Bouville, en faveur des Grandmontains de Verdelais, portant sur la dîme du pain levée dans les castella de Benauges et Saint-Macaire ${ }^{119}$. Or le même jour, le vicomte de Bezeaumes, Bernard de Bouville, était aux côtés du roi lors de la confirmation des privilèges de La Sauve. En confirmant les donations de Verdelais, du vivant du principal intéressé et peut-être en sa présence, Richard affirmait sa suzeraineté sur cette seigneurie, de Benauges à Saint-Macaire. Un sirventes de Bertran de Born, daté des années 1185-1187, évoque justement le contrôle de Benauges ou Bezeaumes "près de Bordeaux ", sans apporter plus de détails ${ }^{120}$.

En même temps, Richard conservait à la ville de Saint-Macaire une attention particulière. En mars 1190, depuis Chinon, il adressa une demande de prêt aux prud'hommes de Saint-Macaire, tout en reconnaissant *qu'ils n'étaient pas ses hommes * (cum homines nostri non sitis), puisqu'il s'agissait visiblement des bourgeois du bourg prieural ${ }^{121}$. Cinq ans plus tard, Mercadier, le chef de ses mercenaires séjournait dans la place ${ }^{122}$.

En Entre-deux-Mers, Richard put completer son dispositif avec la mise en place du castellum du Cros, dans la paroisse de Loupiac. Le 28 avril 1196, le roi donnait ce castellum à un de ses fidèles, Beaudouin de Cassel, contre hommage et servitium. Cette forteresse avait été édifiée dans une paroisse qui, d'après l'enquête de 1237, était « au roi » et qui constituait avec ses voisines Cadillac et Sainte-Croix du Mont une sorte d'avancée de la directe vers la Garonne et Saint-Macaire, au contact de la seigneurie de Benauges ${ }^{123}$. Mais Le Cros n'avait pas seulement vocation à surveiller cette seigneurie: avec Barsac qui lui faisait face, et qui allait être au milieu du XIII' siècle le siège d'une prévôté royale, le roi-duc verrouillait les deux rives de la Garonne.

\footnotetext{
115 J.-P. Migne, (voir note 118) col. 1020-1021.

119 R. de Rouvray, (voir note 106) p. 281, d'après la Chronica coenobionum du prieur Jean Itier de Grandmont, 1492. D'après la même source, Richard se serait rendu à Verdelais quelques jours plus tard, aurait pris les religieux sous sa protection, donné 300 pièces d'or pour agrandir la chapelle, pour une messe hebdomadaire à son intention et faire brùler un cierge pendant la durée de la croisade: $R$. de Rouvray. (voir note 106) p. 34.

${ }^{120}$ G. Gouiran, (voir note 11) p. 518, P Molt m'es descendre car col: Pos lo coms Richarts mais vol, Briermes sai pres Bondel, Qe Conhat ni Mirabel (...) =.

${ }^{221}$ Cart. Ste Croix, (voir note 9) n'72, - Ricaldus (...) dilectis sui probis hominibus de Sancto Machario. Quoniam confidimus de vobis sicut de fidelibus at dilectis nostris mandamus vobis at mgamus quatinus accomodetis nobis $I$. libras de Burdegala (...). Hoc autem non mandamus vobis quia hoc facer debeatis cum homines nostri non sitis, sed quia de vobis confidimus at quia opus habemus ut nobis in hoc subveniatis. -

${ }^{122}$ L. Landon, (voir note 106) p. 101.

${ }^{13}$ P.C.S.M., p. 131, - Item domnus Henricus vendidit (...), viccomitissa de Benauias parrochias de Cadilhac, de Lopiac et Sancte Crucis de Monte, homines domni ngis francos at ab omni servitute liberos. -
} 


\section{Au nord de la Dordogne}

Au nord de la Dordogne, les éléments du dispositif richardien n'apparaissent pas aussi distinctement. On sait par une lettre de Jean Sans Terre du 28 juillet 1200 que Richard avait autrefois confirmé la sauveté de l'abbaye de Guitres et peut-être déjà placé cet établissement sous sa garde et protection ${ }^{124}$. Or Guitres est située sur la vallée de L'Isle, entre les deux vicomtés les plus septentrionales de notre région, Fronsac et surtout Castillon, dont le vicomte Pierre III, participa au soulèvement de 1182-1183 (voir infra).

Il est également possible que Richard se soit intéressé à Saint-Emilion. L'autorisation par Jean Sans Terre aux bourgeois de Saint-Emilion de fonder une commune en juillet 1199 montre que le roi n'était pas absent d'un lieu où jusqu'alors les seuls seigneurs connus étaient le vicomte de Castillon et l'archevêque de Bordeaux $^{125}$.

\section{Richard « seigneur de Bordeaux *}

Quoique Bordeaux n'ait pas toujours occupé une position centrale dans les préoccupations de Richard, celui-ci y séjourna assez souvent pour que Bertran de Born l'appelle a seigneur de Bordel - (1185-1186 $\left.{ }^{126}\right)$. Il confirma les possessions des établissements religieux dont les dépendances représentaient un intérêt stratégique. La première, l'abbaye bénédictine de Sainte-Croix de Bordeaux, reçut ainsi deux confirmations de ces possessions assorties d'une protection, portant nommément sur ses prieurés de Soulac, Le Taillan, Macau et Saint-Macaire (1174 et $1182)^{127}$. La cathédrale Saint-André de Bordeaux en 1186 eut confirmation de ses privilèges, dont le droit de percevoir des revenus de la monnaie de Bordeaux et des péages situés entre l'entrée de l'estuaire de la Gironde (Mortagne) et Langon. Richard confirma également les possessions du prieuré Saint-Martin du Mont-Judaïque, situé au nord-ouest de Bordeaux et accorda aux Grandmontains une importante dotation au sud de la ville $\mathrm{e}^{128}$.

Richard puisait à Bordeaux, dont il était seigneur pour l'essentiel, ainsi que dans les terres de la directe environnante de quoi rétribuer ses fidèles ${ }^{129}$. Ainsi entre 1185 et 1189 , donna-t-il contre cens et esporle une place à bâtir à Bordeaux, dans le quartier de la Rousselle, à Pierre d'Auzac ${ }^{130}$. Plus tard, un certain Chitres, un arba-

\footnotetext{
124 Rot. chart., (voir note 96 ) p. 73.

${ }^{13}$ F. Boutoulle, - Le ban de l'archevèque de Bordeaux à Saint-Emilion (1079-fin du XI1' siècle) -, Vignes, vins $e t$ vignerons, de Saint-Emilion et d'ailleurs, LII' Congrès d'études régionales de la Fédération historique du Sud-Ouest tenu à Saint-Emilion le 11 et 12 septembre 1999, Bordeaux 2000, p. 42-56.

13 Ed.G. Gouiran, (voir note 11) n*21 (1185-1186).

in Cart. Ste-Croix, (voir note 9) n*4 (1174); n'34 (1182).

Is A. D. Gironde, G. 75 p. 1, f. 7 v. (confirmation d'Edouard $\mathrm{I}^{\mathrm{r}}$ ). - Patent rolls of the rign of Henri III pre senved in the P.R.O., A.D 1225-1232, - p. 390, (confirmation en 1232), portant sur les paroisses de Léognan, Villenave, Bègles au sud de la ville. Nous n'avons pas pu localiser l'église Notre-Dame de Cornelie, à qui Richard, duc d'Aquitaine, confirma une donation d'Aliénor portant sur des aumónes à Bordeaux: Róles Gascons, éd. F. Michel, t. I, Paris, $1885, \mathrm{n}^{\circ} 1047$.

13 Les seules partie de la ville qui échappaient à la juridiction ducale étaient la sauveté de saint-André au sud-ouest de la cité antique et les sauvetés suburbaines de Sainte-Croix de Bordeaux, au sud, et celle de Saint-Seurin, au nord ouest.

${ }^{190} \mathrm{Rec}$. feod., (voir note 99) n495, et A.H.G., (voir note 39) L. III, p. 18-19: acte non daté, rédigé à La Réole, entre 1185 (Robert de Montmirail sénéchal de Poitou) et 1189.
} 
létrier qui avait été émasculé par les hommes du comte de Toulouse, reçut de Richard et de son neveu Othon de Brunswick, en 1196 ou 1197, les terres et les hommes de Bègles, Corbiac, Combes et Laforêt ${ }^{131}$. A une date indéterminée, Richard donna une placia à Bordeaux à Rufat et Raimond Béguey, deux représentants d'une des familles bourgeoises les plus en vue de la ville ${ }^{132}$. Avant qu'il n'accède au trône, Richard accorda aux moines de Grandselve une franchise de coutume pour une embarcation transitant sur la Garonne et le droit de prendre un muid de sel sur le salin de Bordeaux ${ }^{133}$; il en informa ses baillis, le « sénéchal de Bordeaux * et ses " fidèles citoyens de Bordeaux * qui, comme à Agen, devaient avoir affermé ces $\operatorname{taxes}^{134}$.

\section{Limitation du recul de la suzeraineté ducale}

Vis-à-vis du baronnage gascon l'activisme de Richard eut quelques résultats tangibles.

Une faible participation du baronnage gascon au soulèvement de 1183

Deux sources peuvent nous permettre de mesurer l'implication du baronnage gascon dans le soulèvement de 1182-1183. Bertrand de Born, principal instigateur de la ligue contre Richard, regrettait dans le sirventes $\approx$ Pois Ventadorns e Comborns ab Segur Dompna, Puois de mi no-us cal " l'attitude attentiste du comte d'Armagnac, des vicomtes de Béarn - qualifié de chef des Gascons- Marsan, Lomagne et Dax (entre juillet et fin 1182) ${ }^{135}$. Geoffroy du Vigeois, signalait la rébellion du vicomte de Castillon et de son frère, Olivier de Chalais ${ }^{136}$, sans rien évoquer pour les premiers cités. Ces deux témoignages suggèrent donc une faible implication du baronnage gascon dans le soulèvement de 1183 .

En 1186, les barons de la Gascogne septentrionale suivirent le duc en Quercy: le traité d'alliance signé à Najac entre Richard et Alphonse II d'Aragon fut passé devant quelques uns des plus importants seigneurs de l'Agenais, du Bazadais et du Bordelais ${ }^{137}$. Le duc avait alors acquis une flatteuse réputation: une charte de

isi B. Cassagne, - Les premiers seigneurs du Tinh -, Mémoire de Guyenne, n"2, 1991, p. 2, d’après P.R.O, C. $52 / 53, n^{\circ} 23$.

${ }^{19}$ Rot. Chart., (voir note 96) p. 4 b; Róles Gascons, t. II, (voir note 107) n'1588 (confirmation de Jean sans Terre).

${ }^{13}$ Rot. chart., (voir note 96) p. 62 (confirmation de Jean) ; C. Devic, J. Vaissette, Histoirr gínérale du Languedor, t. VIII, 2, col. 1850.

is B.N. lat. 11010, f. 144 vo; B.N. Doat, 80, f. 309. Communiqué par N. Vincent.

15 Ed. G. Gouiran, (voir note 11) p. 189, - Si-l rics vescoms que es caps dels Gascos, a cui sórten Bearns e Gavardans, en Veians, si-s vol, en Bernaros, el srigner d'Aics e cel sui e Marsas =; A. Richard, (voir note 28) p. 212.

${ }_{15}$ R.H.F.,(voir note 2) t. XVIII, p. 213 (1182), - Olivarius, frater Prtri vicecomiti Castellionensis munivit Cale sum contra ducem. - Ses chevaliers avaient enlevé le corps de sainte Ancilde et l'auraient transporté dans la chapelle du château de Tulle, A. Richard, (voir note 28) p. 208 d'après H. Duplès-Agier, Chronique de Saint-Martial de Limoges, Paris, 1874, pp. 60-61 et LaввÉ, Novr Bibilioteca man. II. p. 332. Bertran de Bom évoque dans un de ses sinventes la beauté de la - vicomtesse de Chalais =, éd. G. Gouiran, (voir note 11) p. 113.

${ }_{15}$ R. Benjamin, = A Forty Years War: Toulouse and the Plantagenêt, 1156-96 -, Historical Research, t. 61, 1988, p. 283-284, Barcelone, Arxiu de la Corona d'Aragó, Cancelleria, Pergamins d'Alfons ler, n'387: Etienne de Caumont, Guillaume Raimond de Pins, Guillaume de Blanquefort, Pierre de Lamotte, 
1186 du cartulaire de La Réole est datée, comite vero Pictavensi probissimo, milite Ricardo ${ }^{138}$.

Une suzeraineté ducale inégalement renforcée

Les efforts de Richard en Gascogne visaient aussi à renforcer la suzeraineté ducale. Ainsi pouvait-on voir, le 3 février 1190 à La Réole à la cour du nouveau roi, la fine fleur du baronnage du Bordelais, du Bazadais, de l'Agenais, de la Lande et du Quercy (fig. 2) ${ }^{139}$ avec, en tête, le comte d'Armagnac et de Fézensac Bernard IV (1160-après 1193) que Richard avait aidé en 1182, puis Bernard de Bouville, vicomte de Bezeaumes, et Gaston VI, vicomte de Béarn ${ }^{1 * 0}$. Richard a également limité la vassalité du Béarn vis-à-vis de l'Aragon : I'hommage de Gaston VI rendu à Alphonse II en 1187 à Huesca, comporte une réserve des droits du duc d'Aquitaine (« je vous aiderai contre tous les hommes, sauf contre le comte de Poitiers " ) ${ }^{141}$. Dix ans plus tard, lorsqu'Aliénor se rendit à Bordeaux le $1^{\mathrm{e}}$ juillet 1199, Gaston VI de Béarn assistait à sa cour, ainsi qu'Arnaud Raimond, le vicomte de Tartas ${ }^{142}$.

Cependant, cette politique était incomplète. Au mois de mars 1196, à Olite, le vicomte de Tartas, Arnaud Raimond, rendait hommage au roi de Navarre, Sanche le Fort; le vicomte s'obligeait à faire la paix ou la guerre selon la volonté de son nouveau seigneur « contre tout homme du monde, même contre le roi d'Angleterre ou le vicomte de Béarn ${ }^{143}$. Le roi de Navarre marquait ainsi un nouveau point dans le

Gautier du Fossat, Guillaume Séguin de Rions, Hugues de Pardailhan, Gaubert de Fumel, le vicomte de Castillon, Bernard de Bouville, Séguin de Balens, Othon de Caseneuve, Raimond Bernard de Montpezat, Gaubert Flame et Hugues de Pujol.

is Cart. La Rrole, (voir note 9) n"105.

15 G.C.S.M., n'1106, apparaissent d'abord les ecclésiastiques: Géraud, archevèque d'Auch, Adémar évêque de Périgueux, Bertrand évéque d'Agen, Gaillard évếque de Bazas, Arnaud abbé de Clairac, Etienne abbé de La Chaise-Dieu, Guillaume abbé de Brantôme, Bertrand abbé de Moissac et Aimon abbé de Cadouin; puis les laies, Hélie de Celles, sénéchal de Gascogne, Henri flius ducis Gasconie (sic. pour Saxe), Bernard comte d'Armagnac, Bernard vicomte de Bezeaumes, Gaston vicomte de Béarn. Pierre vicomte de Castillon, Guillaume Amanieu de Fronsac, Aiquelm Guillaume de Marsan, Amanieu d'Albret, Amauvin de Blanquefort, Pierre de Lamotte, Aiquelm Guillaume de Lesparre, Geoffroy de Pons son frère Renaud, Gérard de Bourg, Hélie Roil, Guillaume de Gourdon, Etienne de Caumont. Aner Sanche son frère, Guillaume Raimond de Pins, Bertrand de Fumel, Gérard preboides de Montcuc. Nicolas le chapelin du roi, Milon son aumonier, Robert de Osavilla, et Roger Marechal. La liste fournie par la confirmation des privilèges de Saint-Sever le 3 février est moins complète, P. D. du Buisson, (voir note 98) p. 203 (Géraud archevèque d'Auch, Adémar de Périgueux, Bertrand d'Agen, Arnaud abbé de Clairac, Hélie de Celle, Bernard comte d'Armagnac, Gaston vicomte de Béarn, Guillaume Amanieu de Fronsac, Arnaud Guillaume de Marsan, Amauvin de Blanquefort, Pierre de La Motte, Aiquelm Guillaume de Lesparre, Guillaume Raimond de Pins, Gerard de Mones, Milon aumônier du roi ; L. Landon, (voir note 106) p. 25.

169 J. de Jaurgain, (voir note 16) p. 557.

11 P. Tucoo-Chala, (voir note 20 ) p. $151, \mathrm{n}^{*} 8$.

18 E. du Laura, (voir note 7) t. I, p. 120: Hélie, archevẻque de Bordeaux, Henri, évêque de Saintes, Bernard évéque de Lectoure, Etienne doyen de Bordeaux, Gaston, comte de Bigorre et vicomte de Béarn, Raimond Bernard de Rouman, sénéchal, Guifard de Didone, Amauvin de Blanquefort, Ayquelm Guillaume de Lesparre, Pierre de Lamotte, Humbert de Foix, Arnaud Raimond de Tartas, Raoul de la Faye, Geoffroy Rudel de Blaye, Guillaume Séguin de Rions.

is J. de Jaurgain, (voir note 16) p. 609-610. 
recouvrement d'une ancienne suzeraineté au nord des Pyrénées ${ }^{1+4}$. Pourtant, la vicomté de Tartas ne se détachait pas complètement du roi-duc: Arnaud Raimond continua de fréquenter la curia ducale (1199-1200) et à servir le Plantagenêt dans sa politique régionale $e^{145}$. L'hommage de 1196 recouvrait certainement les seigneuries de Mixe et d'Ostabarret que le vicomte de Tartas venait d'acquérir par son mariage avec Navarre, vicomtesse de Dax, dame de Mixe et d'Ostabarret. Dans le même temps, le vicomte de Soule suivait la même voie: le vicomte Raimond Guillaume se trouvait en effet à Olite en mars 1196, pour faire juger par Sanche le Fort un différent avec le vicomte de Béarn, Gaston VI ${ }^{146}$.

Pour Richard, les hommages des vicomtes de Tartas et de Soule n'avaient peutêtre pas la portée de celui du vicomte de Béarn avant 1187: ils profitaient en effet à un beau-frère qui avait aidé ses sénéchaux à vaincre les séditions du baronnage en 1192 et $1194^{147}$. C'est pourquoi ces transferts de suzeraineté avaient certainement été négociés entre les deux hommes.

\section{L'affermissement de la puissance ducale}

Au delà du souci de contrôler ces nombreux points d'appui ou des seigneuries plus ou moins hostiles, la politique d'Henri et de Richard en Gascogne s'est traduite de manière plus diffuse par un alourdissement de la fiscalité et de l'autorité ducales. Nous pouvons observer ce phénomène en Bordelais où, pour l'instant, la documentation a été plus exhaustivement dépouillée.

\section{Le souci de la paix publique}

Richard, pendant son règne, s'est attaché à ce que la paix publique fût respectée en Gascogne. A Bayonne, si l'on en croit la * charte des malfaiteurs ", lo rey Richart aurait édicté entre 1195 et 1197 , une série de dispositions coutumières sur la répression des crimes et des délits commis dans la cité et sur le territoire de la vicomté $e^{148}$.

\footnotetext{
"En 1191, Sanche VT avait été en mesure de donner en dot pour sa fille Bérengère les châteaux de SaintJean-Pied-de-Port et de Roquebrune; il les avait encore en 1198: J. Gillingham, (voir note 74) p. 360.

is Le 30 janvier 1200. Jean mande à Arnaud Raimond de Tartas et à ses autres agents dans la région d'attendre les instructions que leur amènerait le sénéchal (A. Richard, voir note 28, p. 368; Rot. chart. voir note 96, p. 58b). Le 29 janvier 1200 , Jean annonce à ses émissaires envoyés ad pacificandam Vasconie, qu'il ne ferait pas la paix avec Gaston, vicomte de Béarn, si son adversaire Amaud Raimond, vicomte de Tartas n'y était pas compris (Rot, chart., voir note 96, p. 58 ). Son successeur, Raimond Arnaud, vicomte de Tartas et de Dax reçoit des mandements d'Henri III en 1242, tout en prètant hommage lige à Thibaud $I^{e r}$ de Navarre pour les terres de Mixe et Ostabarret en 1247 (J. de Jaurgain, voir note 16, p. 611).

$14 \mathrm{~J}$. de Jaurgain, (voir note 16) t. II, p. 467.

167 A. Richard, (voir note 28) p. 280, 293.

${ }^{14} \mathrm{~J}$. Balasque, (voir note 52) $\mathrm{n}^{\circ}$ XIV, p. 419, d'après Archives de Bayonne, AA-II,p. 8, * Asso que sec de jus part son les justiciis et condempnations deux maufotors, fole at autryadas aux de Baione per lo my Richart, $m$. $d$ 'Angleterr, $d a b$ sa voluntat... . . Texte en gascon sans appareillage diplomatique et non daté. L'établissement a été pris sous le témoignage de Guillaume Bertrand, évêque de Dax (1168-1203), Bertrand de Lescar évèque de Bayonne (1186-1213), - Jaufre de Batele, senescau de Potau et de Gasconher- (Geoffroy de Celles), des preux chevaliers de la terre, des prud hommes de Bayonne et de - tout l'autre peuple de la ville =. Le sénéchalat de Geoffroy de Celles permet de dater les dispositions de 1195-1197: cart. Ste-Croix, (voir note 9) n`29: A. Richard, (voir note 28) t. II, pp. 308, 310, 311, 317; F. Boutoulle, - Hélie de Malemort, archevêque de Bordeaux: un prélat politique au service de Jean sans Terre (1199-1207) =, Revue historique de Bondeatux at du département de la Gironde, troisième série, n4, 2004, p. 7-23, note 57.
} 
En Bordelais, il utilisa l'ancien mouvement de la Paix de Dieu que les chefs des principautés méridionales venaient de reprendre à leur compte ${ }^{149}$ : entre les mois de mai et décembre 1197, Richard fit édicter un statut de paix (voluntate et praecepto domini $R$. regis), avec le conseil de l'archevêque Hélie de Malemort, du sénéchal Geoffroi de Celles, des barons, chevaliers et prud'hommes du pays, pour marquer peut-on penser la canonisation de Gérard de Corbie survenue l'année même. Ses dispositions, jurées par un serment commun, devaient être respectées pendant 10 ans; un impôt de paix, appelé « commun du roi ", ayant le cheptel pour assiette, devait ètre levé pendant 7 ans ${ }^{150}$.

En ces deux occasions (nous ne savons pas directement si Richard est venu sur place), le roi s’appuya sur les communautés urbaines avec qui il était déjà en relation par la firma burgis. A Bayonne, la «charte de malfaiteurs * évoque un conseil de la ville et une commune ${ }^{151}$. En Bordelais, les structures communautaires prévues par la paix de 1197, à la demande des « prud'hommes * du pays, ont certainement renforcé le rôle fiscal des communautés urbaines et leur structuration. Les a jurats * de Bordeaux, attestés en 1200 dans un mandement de Jean Sans Terre, avaient la main sur les revenus des péages, du salin et des zones restées dans la directe environnante ${ }^{152}$.

\section{Alourdissement de la fiscalité et des exigences militaires}

La documentation bordelaise révèle de manière indirecte l'alourdissement de la fiscalité ducale pendant les règnes d'Henri II et de Richard. Les « petites coutumes * (parvas consuetudines) levées à Bordeaux sur le vin et dont Aliénor affranchit les Bordelais le $1^{e r}$ juillet 1199, paraissent avoir été mises en place par Richard puisqu'elles étaient qualifiées d' « inhabituelles et inusitées * (inauditis et indebitas) ${ }^{153}$. Une a coutume sur l'avoine - était prélevée au moins depuis le règne de Richard: le $1^{\text {er }}$ mai 1206, le roi Jean confirma à Arnaud de Lalande les donations que son père avait reçues du roi Richard, notamment la consuetudo avene $e^{154}$. La maltôte des salines, qui apparaît dans une lettre du 12 octobre 1203, devait être aussi ancienne: Jean confirmait dans ce mandement une franchise d'Aliénor en faveur du camerier de l'archevêque de Bordeaux lui permettant de transporter tous les ans, 5 muids de sel, de Bordeaux à Langon, « sans coutume, ni maltôte * (absque omne consutuetudine et malatolta $)^{155}$.

\footnotetext{
16 T. N. Bisson, - The Organised Peace in Southern France and Catalonia, ca. 1140-ca. 1233 -, American historical miru, 1977, t. 82, p. 290-311.

1se Cart. St-Seurin, (voir note 12) n' 204; F. Boutoulle, (voir note 41) p. 448-453.

ist J. Balasque, (voir note 52) n'XIV, p. 420, - affermat suber lo sagrementz dou cosselh de Baione $t$ de tot lo comunau. :

${ }^{152}$ F. Boutoulle, (voir note 41) p. 612-620. La commune et le maire de Bordeaux n'apparaissent pas dans la documentation avant 1206. Notons que la mème année 1197 voit l'apparition du consulat d'Agen. sous suzeraineté toulousaine: Chartes, première śrie, n'II; S; Baumont, s.d., Histoine d'Agen, Toulouse, 1991. p. 47.

${ }^{15 s}$ Ces taxes s'appèlent luch, vinada, lo bech prepositi et lo bech deu mess vindemiandis, cf. Livne des coutumes, Archives municipales de Bordeaux, éd. H. Barckhausen, Bordeaux, 1890, nXLV, p. 437 (1" juillet 1199 ) ; la transcription du Livre Velu de Libourne remplace - lo bech deu megs vindemiandis - par - de vineis vindemiandis $=$.

${ }^{14}$ Rotuli litterarum patentium in turri Londinensi asservati, (voir note 113), vol. 1, pars 1, 1201-1226, p. 63.

iss Rot. chart., (voir note 96) p. 112 p. 74 b.
} 
Les premiers Plantagenêts ont aussi certainement introduit une réserve sur le droit de garde des héritiers et des biens d'un noble décédé ${ }^{156}$. Entre 1165 et 1181 , Pierre de Bordeaux, donnant en sa dernière extrémité, pouvait encore confier la garde de sa domus, de son épouse et de ses fils à deux milites de sa famille ${ }^{157}$. Par la suite cette possibilité fut considérablement limitée. Dans l'enquête de 1237, on rapporte comment le roi Richard avait jadis donné en mariage la domina de Corréjan à un de ses fidèles, un certain Hugon $\operatorname{Los}^{158}$. D'après les dépositions de la même enquête, cette coutume « venait d'Angleterre * et fut plutôt mal accueillie (corruptelam Anglie introducens quasi per consuetudine in terra $)^{159}$.

Le service militaire des nobles (bien attesté par les premiers mandements de Jean Sans Terre $)^{160}$ a peut-être été remplacé par une taxe évoquée par l'enquête de $1237^{161}$ qui rappelle l'écuage anglo-normand. Vis-à-vis des plus humbles, les premiers Plantagenêts ont aussi exigé un service armé: les coutumes des cives de Bayonne, confirmées par Richard entre 1170 et 1172, faisaient obligation de suivre le sénéchal in expeditio pour tirer vengeance d'un outrage fait à un officer ou à un des hommes du comte, sous peine de devoir payer 6 sous $^{162}$. En confirmant les privilèges de Saint-Sever, Richard s'interdisait de requérir les hommes de l'abbaye en " ost ou en chevauchée ${ }^{163}$. En Bordelais, il semble même que cette exigence se soit alourdie. En effet, la confirmation des privilèges de La Sauve-Majeure du 3 février 1190 montre que les bourgeois de La Sauve et les hommes de l'abbaye avaient été, par le passé, requis par les officiers du roi à l'exercitus ${ }^{164}$; or cette clause n'apparaît pas dans la confirmation des mêmes privilèges passée par Henri II en décembre $1156^{165}$. Si la prestation n'a pas été imposée dans l'intervalle, du moins fut-elle suffisamment alourdie pour que les moines de La Sauve ressentissent le besoin d'en être explicitement libérés ${ }^{166}$.

156 P.C.S.M., p. 130, terram at filios vel filias decentium nobilium occupat.

157 Cart. St-Seurin, (voir note 12) n"136 (1168-1181), - presentibus uxore sua Marchiesa et predicto Matheo ot Rufato canonicis duobusque militibus (...) quibus curam domus uxoris a filionum commitebat. =

15. P.C.S.M., p. 132, - modus alienationis fuit rex Ricardus dedit cos Hugoni Los, cum ci dedisset in uxorm dominam de Conrjan, quo mortuo sine herde, uxor gius nupsit cum Willelmus Amaldi de la Mota. -

is P.C.S.M, p. 130, - item comuptelam Anglie introducens quasi per consuctudine in terra, terram et filios vel filias decedentium nobilium occupat, et tamdiu detinet, donec se ndimant vel nubant cui ipse voluerit at cui non deceat nubere. - Aussi, en 1214, Pierre de Bordeaux doit-il débourser 20000 sous poitevins pour avoir la garde de I'honneur de Blanquefort et de l'lle, celle des droits de Guillaume de Blanquefort à Bourg-sur-Mer et pour pouvoir marier sa fille, Assalhide, héritière de cet honor; A.H.G., (voir note 39 ) t. 4, n"IX, p. 12 (1214), et Rot. chart., (voir note 96) p. 118 (6 juin 1214).

160 Rot. litt. pat., (voir note 113) p. 3, 36 b; Foedera, contentiones, literrae et cujuscumque generis acta publica, éd. T. Rymer, vol. I- III, London, 1821, p. 155.

${ }^{161}$ P.C.S.M., p.128, - quicumque tenet res que debent exercitum vel unius militis vel duorum, nisi privilegio tueatur, secundum partem quam tenet facit partem expensarum ei qui facit axcritum domini rgis, -

${ }_{16} \mathrm{~J}$. Balasque, (voir note 52) n"XII : - Et per quicumque senscallus meus ert, per totam terram meam at ultra quantum possit ei conductum prestare debebit. Practerea si aliquis senescallo vel hominibus meis injuriam fecerit, ad injuriam vindincandam sequentur senescallum in expeditionem; quod si facere noluerint, quisque remanensium VI solidos prestabit. Si vero senescalluus, accepta pecunia, nulla injuria sibi vel suis illata, axpeditionem facere voluerit, nullatenus eum sequentur. :

${ }^{165}$ P. D. du Buisson, (voir note 98) p. 200-204. Disposition reprise des privilèges des ducs Guillaume Sauche et Bernard Guillaume.

164 G.C.S.M., n`1106, - vel homines eorum aliquis ad expeditionem aliquam seu ad arma aliquatenus compel. lat. =

16 A.D. 33, H 12; Recucil des actes d'Henri II, (voir note 7) n'XXV, p. 118.

${ }^{166}$ D'après les coutumes de l'Entre-deux-Mers, les habitants de cette région devaient participer à l'exercitus avec les communia civitatum a burgorum, P.C.S.M., p. 130. 


\section{Les officiers ducaux en Gascogne}

L'appesantissement de l'autorité ducale sur un territoire où le duc était souvent absent pose le problème de ses relais sur place, de leur nombre et de leur efficacité.

Initialement, la Gascogne avait été intégrée au ressort des sénéchaux d'Aquitaine. Quand les coutumes de Bayonne, accordées par Richard en 1170-1172, faisaient obligation de suivre le sénéchal à travers toute la terre du duc, aucun ressort territorial ne lui était affecté $e^{167}$. De fait, le sénéchal du Poitou Foulque de Matha a suivi Richard à Dax et Bayonne en 1172. Il faut attendre les années 1180 pour qu'un office de sénéchal de Gascogne soit explicitement attesté (voir tableau suivant) ${ }^{168}$.

Les sénéchaux de Gascogne de 1182 à 1200

\begin{tabular}{|l|l|}
\hline Dates repères & Sénéchal \\
\hline 1182 & Pierre Arb. \\
\hline 2 février 1190 & Hélie de Celles \\
\hline $1195-29$ décembre 1198 & Geoffroy de Celles* \\
\hline 31 mars $1199-\mathrm{I}^{\text {er }}$ juillet 1199 & Raimond Bernard de Rouvignan \\
\hline 29 janvier 1200 & Brandin \\
\hline
\end{tabular}

On relève le premier «sénéchal de Gascogne ", Pierre Arb. (sic) aux côtés du sénéchal de Poitou, Guillaume Capon dans une confirmation de Richard en faveur de Sainte-Croix de Bordeaux, datée de $1182^{169}$. La séparation des deux fonctions, suggérée par cette charte, n'était cependant pas définitive. Alors que dans les années qui suivent, on ne rencontre plus de sénéchal de Gascogne, certains sénéchaux de Poitou ont retrouvé des attributions au sud de la Gironde : ainsi en 1185 , à La Réole, le sénéchal de Poitou, Robert de Montmirail assista à une donation de Richard concernant une maison de Bordeaux ${ }^{170}$.

En 1190, les sénéchaussées de Poitou et de Gascogne étaient à nouveau disjointes. Le 3 février de cette année, depuis La Réole, Hélie de Celles, senescallus Gasconie, assistait en compagnie de nombreux prélats et barons de la Gascogne à la confirmation des privilèges de La Sauve-Majeure par Richard ${ }^{171}$. Cinq ans plus tard, la Gascogne revenait dans les attributions du sénéchal de Poitou: en 1195, le senescallus Pictavie et Vasconie, Geoffroy de Celles, un parent du précédent qui avait suivi Richard en Orient, assisté de l'archevêque de Bordeaux, Hélie de Malemort,

\footnotetext{
${ }^{167} \mathrm{~J}$. Balasque, (voir note 52) n"XII, p. 412; Petit cartulaire de La Satur-Majeure, Bibliothèque municipale de Bordeaux, ms 770 (1237), - tempore predictorum ngum, Henrici et Ricandi, quod unicus aset senescalcus totius terre ngis in archiepiscopatum Burdegalensis a Auxitano. -

${ }^{160}$ Pour compléter les listes de A. Debord, La société lä̈que dans les pays de la charente $X^{*}$-XIr siècles, Paris 1984, p. 402 et de W. W. Shirley, (voir note 78) p. 399-400. Nous avons porté sur le tableau les dates extrêmes de chaque sénéchalat. Les astérisques correspondent à ceux qui ont cumulé les sénéchalats de Gascogne et de Poitou.

16 Cart. Ste Croix, (voir note 9) n"34, - donum istud a concrsio facta et in Crusem, trste me ipro, Guillelmo Caponis senescallo Pictavie at P. Arb. senescallo Guasconie, Augrrio Longuo, prosentibus at mihi assistentibus. -

170 re. frod. (voir note 99) n'495 a. Le texte, reproduit dans une reconnaissance de 1274 , n'est pas daté. Cependant le sénéchalat de Robert de Montmirail s'est déroulé en 1185: A. Richard, (voir note 28) t. II, p. 234, 236, 242, 266, 343.

${ }^{17}$ G.C.S.M, n*1106. L. Landon, (voir note 106) p. 25 . Il était encore le 17 avril 1190 (op. cit., p. 31).
} 
ramena la paix entre le seigneur de Lesparre et l'abbaye de Sainte-Croix, depuis Mont-de-Marsan, in camera regis ${ }^{172}$. Le 25 mai 1196, il était «sénéchal de Gascogne ${ }^{173}$, mais en 1197, lorsque fut établie la paix du roi en Bordelais, Geoffroy cumulait les deux fonctions $^{174}$. Il était encore sénéchal le 24 juin $1198^{175}$ et le 29 décembre $1198^{176}$. Il perdit ses attributions peu après: le 31 mars 1199 , le sénéchal de Gascogne était Raimond Bernard de Rozinan ou Rovignan jusqu'au premier juillet 1199 au moins ${ }^{177}$.

Des sénéchaux ayant des attributions plus limitées apparaissent exceptionnellement à Bordeaux et à Agen, dans les franchises accordées à Grandselve. Ces sénéchaux, que l'on ne retrouve pas ultérieurement dans la documentation, devaient veiller à la levée des coutumes sur le traffic fluvial.

Comme le montrent les adresses de leurs actes, les Plantagenêts ont également introduit les justiciers en Gascogne (judiciarii ou justicii) ${ }^{178}$. Les seuls que nous connaissions, Aimeric Brun et Long Auger (tunc temporis domini Richardi comitis Pictave, in Vasconiam mandatoribus et justiciarii) ont eu à juger un conflit entre le prieur de La Réole, Arnaud, et Sanche de Corbian, portant sur une dîme $(1180)^{179}$. Aimeric Brun est encore cité en 1182 aux côtés du sénéchal de Gascogne, Pierre $A r b^{180}$. Ces premiers justiciers de Gascogne ressemblent fort à ces juges itinérants, cheminant par binôme ou trinôme en Angleterre et systématisés en Angleterre par l'assise de Northampton de $1176^{181}$. Cependant, au delà de la mission arbitrale, la documentation gasconne ne révèle pas l'ampleur de leurs attributions ${ }^{182}$. Ces juges itinérants n'ont pas non plus constitué un durable instrument de contrôle des officiers locaux, comme en Angleterre. Souffrant de la multiplication des prévôtés royales, au début du $\mathrm{XIII}^{e}$ siècle, les justiciers ont peu à peu été cantonnés à un modeste ressort territorial pendant que leurs attributions perdaient de leur substance; vers 1230 les justiciers n'était au plus que des auxiliaires de justice dotés de pouvoirs de police $^{183}$.

\footnotetext{
17 Cart. Ste Croix, (voir note 9) n'29; L. Landon, (voir note 106) p. 50 et 60.

${ }^{173}$ L. Landon, (voir note 106) p. 113.

17 Cart. St-Seurin, (voir note 12) n'204, - voluntate et precepto domini Ricandi ngis Anglie consilio et assensu domni archiepiscopi et G. de Cellis, senescalli Pictavie at Vasconensis, a baronum et milifum a prudentum hominum pax est constituta . Il était sénéchal de Poitou le $1^{*}$ octobre 1196 et le 4 novembre 1197: L. Landon, (voir note 106) p. 124-130.

${ }^{13}$ L. Landon, (voir note 106) p. 130.

${ }^{17}$ Foxdera, conventiones, littere et cujuscumque genenis acta publica, éd. T. Rymer, R. Sanderson, G. Holmes, La Haye, 1745, p. 71 .

17 E. du Laura, (voir note 7 ) p. 285.

17 Cart. La Ríole, (voir note 9) n"113 (1169-1189), - Richardus, comes Pictavie ngis Anglie filius, omnibus archiepiscopis, piscopiis, senescallis, prepositis, et ceteris justiciariis et ballivis suis ad quos presens scriptum pervenerit, salutem; cart. St-Seurin n"348 (1203), Johannes Dei gratia nx Anglie dux Hibernie, dux Normannie et Aquitanie, comes Andegavie, archiepiscopis, ppiscopiis, comitibus, baronibus, justiciariis, vicariis, prepositis, ministris, $t$ omnibus ballivis at fidelibus suis, salutem. -

is Cart. La Réole, (voir note 9) n'84, - ab codem Aimerico et Longo Augerio a tota curia adjudicata fuit = à propos de la dime et de l'église de Corbian.

150 Cart. Ste-Croix, (voir note 9 ) n* 34.

1n] J. Boussard, (voir note 87 ) p. 494.

12 Ibidem, p. 587.

ins Cinquante ans plus tard, les justiciers agissent sur des ressorts territoriaux plus strictement définis. L'enquête de 1237 rapporte qu'il existait deux * mandators seu citatons $a$ exploratons exressuum = dans la prévôté d'Entre-deux-Mers; le ruisseau du Lubert délimitait leurs deux circonscriptions. Chacun d'eux
} devait dans son ressort rechercher les malfaiteurs. F. Boutoulle, (voir note 41) p. 496-497. 
Pour compléter ces relais, nous avons vu que les Plantagenêts se sont appuyés sur certaines communautés urbaines pour la levée des taxes. Ils ont également compté sur l'épiscopat gascon. Si c'est avec Jean Sans Terre que l'archevêque de Bordeaux Hélie de Malemort a pu révéler la large palette de services qu'il pouvait fournir à un souverain, ce prélat apparaît cependant intégré dès 1195 à l'appareil de contrôle de la région en participant à une cour de justice aux côté du sénéchal Geoffroi de Celles, à Mont-de-Marsan ${ }^{184}$. Son prédécesseur, Bertrand de Montaut (1162-1173), ancien évêque de Lectoure, marcha sur Toulouse en 1164 pour le compte d'Henri $I^{185}$. En 1190, l'archevêque d'Auch, Géraud de Labarthe, et l'évêque de Bayonne, Bernard de Lescar, ont été choisis par Richard pour commander avec d'autres laics la flotte des croisés ${ }^{186}$.

\section{Conclusion}

Compte tenu de l'importance géograhique de l'aire étudiée dans cette première enquête, nous ne doutons pas que des traces de l'action d'Henri II et de Richard dans les sources de la région nous aient échappées ${ }^{187}$. Cependant, quoique forcément lacunaire, le corpus rassemblé livre de suggestives indications. Nous pouvons désormais dépasser la vision d'une Gascogne lointaine et négligée par les premiers Plantagenêts ${ }^{188}$. Certes, les titres comtaux ou ducaux ont été abandonnés; Aliénor elle-même, que l'on a fausement fait naître en Gascogne (à Belin, en Bordelais), n'y avait pas ses séjours préférés ${ }^{189}$; certes encore la Gascogne fut amputée de l'Agenais en 1196 et elle constitua une large partie du douaire de Bérangère de Navarre ${ }^{190}$. Pour autant, Henri et Richard y ont tressé un réseau de points d'appui, fait de châteaux et d'établissements religieux protégés à travers lequel se dessine une politique régionale contre le comte de Toulouse, contre les vicomtes de Béarn et leurs propres réseaux ainsi que vis-à-vis des royautés ibériques.

Si les prédécesseurs des premiers Plantagenêts avaient déjà utilisé les forteresses et les gardes sur les établissements religieux, Henri et surtout Richard ont usé de ces moyens plus systématiquement. De même, les coutumes levées sur le trafic fluvial, que l'on repère dans la documentation depuis le $\mathrm{XI}^{e}$ siècle, ont-elles été alourdies. Par ce biais Richard s'implanta plus fermement sur les sites portuaires et

\footnotetext{
${ }^{154}$ F. Boutoulle, - Hélie de Malemort, archevêque de Bordeaux: un prélat politique au service de Jean sans Terre (1199-1207) -, Revue historique de Bordeaux et du département de la Gironde, Troisième série, $\mathrm{n}^{\circ} 4$. 2004, p. $7-23$

iss R.H.F., (voir note 2) t. XVI, p. 109.

1ss Chronica magistri Rogeri de Hovedene, (voir note 94) p. 36: - et ibi constituit Girardum Auxiensem archiepiscopum at Bernandum piscopum de Baiona et Robertum de Sablun et Ricandum de Camvilla at Willelmum de Forz de Ulenum ductores et constabularios totius navigii sui. =

is Nous ne sommes pas par exemple parvenus à localiser le castellum de Guillaume de Chisi, in Gasconiam, pris par Richard au printemps 1190: Chronica magistri Rogeri de Hoveden, (voir note 94) p. 35 ; J. Gillingham, (voir note 74) p. 193. C'est peut-ètre en Cize.

iss M. Aurell, L'mpire des Plantagenè, 1154-1224, Paris, 2003, p. 212-126.

10 F. Boutoulle, - L'Aquitaine d'Aliénor ", Le Festin, n"51, septembre 2004, p. 89.94.

190 Roles Gascons, t. I. supplément, (voir note 111) 4341; A. Richard, (voir note 28) p. 274, 299; P. Chaplais, - Le traité de Paris de 1259 et l'inféodation de la Gascogne allodiale ", Le Mogen Age, 1955, t. L.XI, p. 133, d'après Dom E. Martene et Durand, Veterum scriptorum et monumentorum amplissima collectio, t. I. Paris, col. 995 ; Dom C. Devic et Dom Vaissette, Histoine générale du Languedoc, t. V1, Toulouse, 1879, p. 174 et 190.
} 
s'affirma dans des cités où les évêques avaient acquis de larges prérogatives publiques (Agen, Bayonne). De manière plus originale, ces coutumes ont surtout permis à Richard de nouer des liens avec les principales communautés urbaines de la région, par la firma burgis. A Agen et Bordeaux le système est avéré avant 1189, à Bayonne et Dax probablement. Richard l'a également imposé à certaines villes et sites de plus modeste importance (Marmande, Couthures, Saint-Emilion certainement). Il renforca ce lien avec les communautés par des franchises ou des chartes de coutumes. Celles de Dax, Bayonne et Agen nous sont parvenues. Mais les traditions de coutumes ducales concernant Marmande, l'Entre-deux-Mers bordelais, Uza, voire La Réole, Couthures ou Bordeaux, suggèrent l'existence d'autres concessions, soit en faveur de communautés vivant en habitat dispersé, soit dans le cadre plus classique d'une ville ou d'un castrum ${ }^{191}$.

En relation directe avec le duc, ces communautés urbaines ou rurales se sont illustrées par des services militaires (Dax, Bayonne). Elles ont également pu se doter d'organes communautaires: nous avons la trace de conseils à Agen, Bayonne et Bordeaux probablement, ainsi que d'une commune à Bayonne. Au seuil de notre terme chronologique, aucune des communautés relevant du roi-duc n'avait atteint le stade de la municipalité avec un maire, mais le mouvement était lancé ${ }^{192}$.

Or, lors des crises de 1204-1206, 1219 et 1224, les communautés urbaines, notamment celles de Bordeaux, Bayonne ou La Réole ont tenu le pays dans la fidélité du roi-duc, alors que baronnage gascon et une partie du haut clergé avaient fait défection. Les liens tissés par Richard eurent donc une large part dans la conservation de la Gascogne sous le règne de Jean Sans Terre. De même, nous avons vu que les barons gascons les plus puissants étaient aussi placés sous suzerainté aragonaise ou navarraise. Le capétien n'a donc pas eu autant de prise sur eux qu'il en eut sur le baronnage normand ou poitevin dans son entreprise de conquête des fiefs continentaux après 1202. Il n'est donc pas nécessaire d'invoquer le mythe d'une * Gascogne allodiale, forgé sur le tard et repris par P. Chaplais, pour expliquer la conservation de ce pays par les Plantagenêts au début du XIII ${ }^{e}$ siècle $^{193}$. La politique de Richard, dont Jean hérita des fruits, a été plus déterminante.

\footnotetext{
191 Sur les privilèges - globaux = consentis par les princes en faveur de communautés vivant en habitat dispersé ou semi dispersé, B. Cursente, Des maisons at des hommes, La Gascogne médiévale ( $\mathrm{X} P$ - $\mathrm{X}_{v} f$ siècle), Toulouse, 1998, p. 290-297. La première mention connue d'une coutume de Bordeaux remonte à 1218 (G.C.S.M., n"1334).

ॠa Les études sur les origines des municipalités de la Gascogne n'ont pas considéré ces préliminaires et se sont trop focalisćes sur les Etablissements de Rouen. Par exemple, pour le Bordelais, C. Bemont, - Les institutions municipales de Bordeaux au Moyen Age -, Renue Historique, L.CXXIII, 1916, t. 123, p. 1-53; ID. - La mairie et la jurade dans les villes de la Gascogne anglaise. Bourg et Blaye, Saint-Emilion et Libourne =, R.H.B., mai-juin 1917, p. 121-133, juillet-août 1917, p. 196-205; Idem, - La mairie et la jurade dans les villes de la Guyenne anglaise: La Réole - Annales du Midi, 1919, p. 1-34; Y. Renouard, Bondeaux sots les rois d'Angletern, Bordeaux, 1965, p. 27-30; M. Bochaca, La banlieue de Bordeaux, formation d'une junidiction municipale suburbaine (vers 1250-vers 1550), 1997, p. 41-45.

is $\mathrm{P}$. Chaplais, (voir note 191) p. 121-137. De nombreux actes des $\mathrm{XI}^{*}$ et XII siècles conservés dans les cartulaires gascons sont datés en référence au règne du roi de France: A. Degert, - Le pouvoir royal en Gascogne sous les derniers carolingiens et les premiers capétiens =, Revue des questions historiques, $t$. XXVIII, Paris, 1902, p. 425-443.
} 


\section{Note sur la carte $n^{\circ} 1$}

Il n'a pas été possible, en l'état de nos recherches, de reconstituer précisément et également les juridictions des principaux seigneurs qui s'opposent aux Plantagenêts à la fin du XII ${ }^{e}$ siècle en Gascogne. Sauf pour le Marsan, dont la thèse de M.-J. Fritz a restitué l'étendue au $\mathrm{XII}^{e}$ siècle, nous sommes servi des travaux présentant ces juridictions au-delà de notre terme chronologique, bien que celles-ci aient certainement évolué entre temps ${ }^{194}$. Ainsi apparait-il, d'après les actions des vicomtes de Dax aux $\mathrm{XI}^{e}$ et $\mathrm{XII}^{e}$ siècle contre le vicomte de Béarn, que leur juridiction devait atteindre la confluence des Gaves de Pau et d'Oloron avant d'être réduite par la suite. De même, les enclaves de la vicomté de Tartas au sud et à l'ouest de Dax, suggèrent une vicomté originale plus étendue qu'elle ne l'est à la fin du XIII ${ }^{c}$ siècle. La vicomté de Castillon, qui recouvre une large part de l'Entre-Dordogne au début du XII ${ }^{e}$ siècle (entre Dordogne et Isle) a par la suite perdu du terrain.

Sur la carte, les délimitations des vicomtés de Tartas, Brulhois, Gabardan et Castillon suivent l'emprise de ces seigneuries à la fin du $\mathrm{XIII}^{e}$ siècle, telles que P. Barnabé les a cartographiées ${ }^{195}$. La Maison d'Armagnac de Ch. Samaran ${ }^{196}$, offre une cartographie de l'Armagnac et du Fézensac au XV siècle. Pour le Béarn, qui avait intégré les vicomtés d'Oloron et de Montanerès dès le $\mathrm{XI}^{c}$ siècle, nous avons utilisé les travaux de P. Tucoo-Chala ${ }^{197}$ et pour la Bigorre, ceux de $\mathrm{M}$. Berthe ${ }^{198}$. Il n'a pas été possible de restituer aussi précisément l'étendue des vicomtés de Bezeaumes et de Dax qui ont été démembrées dans le courant du XIII' siècle; pour cette dernière nous nous sommes appuyés sur l'étendue de la prévôté de Dax à la fin du XIII siècle.

\footnotetext{
194 J.-M. Fritz, Histoin, occupation du sol a peuplement de la vicomté de Marsan des origines à sa rúuion aux domaines de la couronne (1607), thèse de doctorat en histoire médiévale, s.d. J. B. Marquette, Université Michel de Montaigne-Bordeaux 3, 2001; ID., - La vicomté de Marsan : longue construction d'une identité -, Bulletin de la Sociắé de Borda, n*466, 2, p. 133-158; Idem, - La vicomté de Marsan: naissance et fossilisation d'une seigneurie gasconne au Moyen Age =, Annales du Midi, t. CXV1, n'247, juillet-septembre 2004, p. 307-329.

${ }_{195}$ P. Barnabe, Entre noi-duc et roi de France: fidélité ou ralliement du pays gascon (1259-1360), thèse de doctorat en histoire médiévale, s.d. J. B. Marquette, Université Michel de Montaigne-Bordeaux 3, 2003.

$15 \%$ C. Samaran, La maison d'Armagnac au XV' siècle et les dernières luttes de la féodalité dans le Midi de la France, Paris, 1907.

197 C. Desplat et P. Tucoo-Chala, Atlas Histonique du Béam, Pau, 1980.

19 M. Berthe, Le comté de Bigone, un milieu nural au Bas Mogen Age, Paris, 1976.
} 
FRÉDÉRIC BOUTOUL.LE.

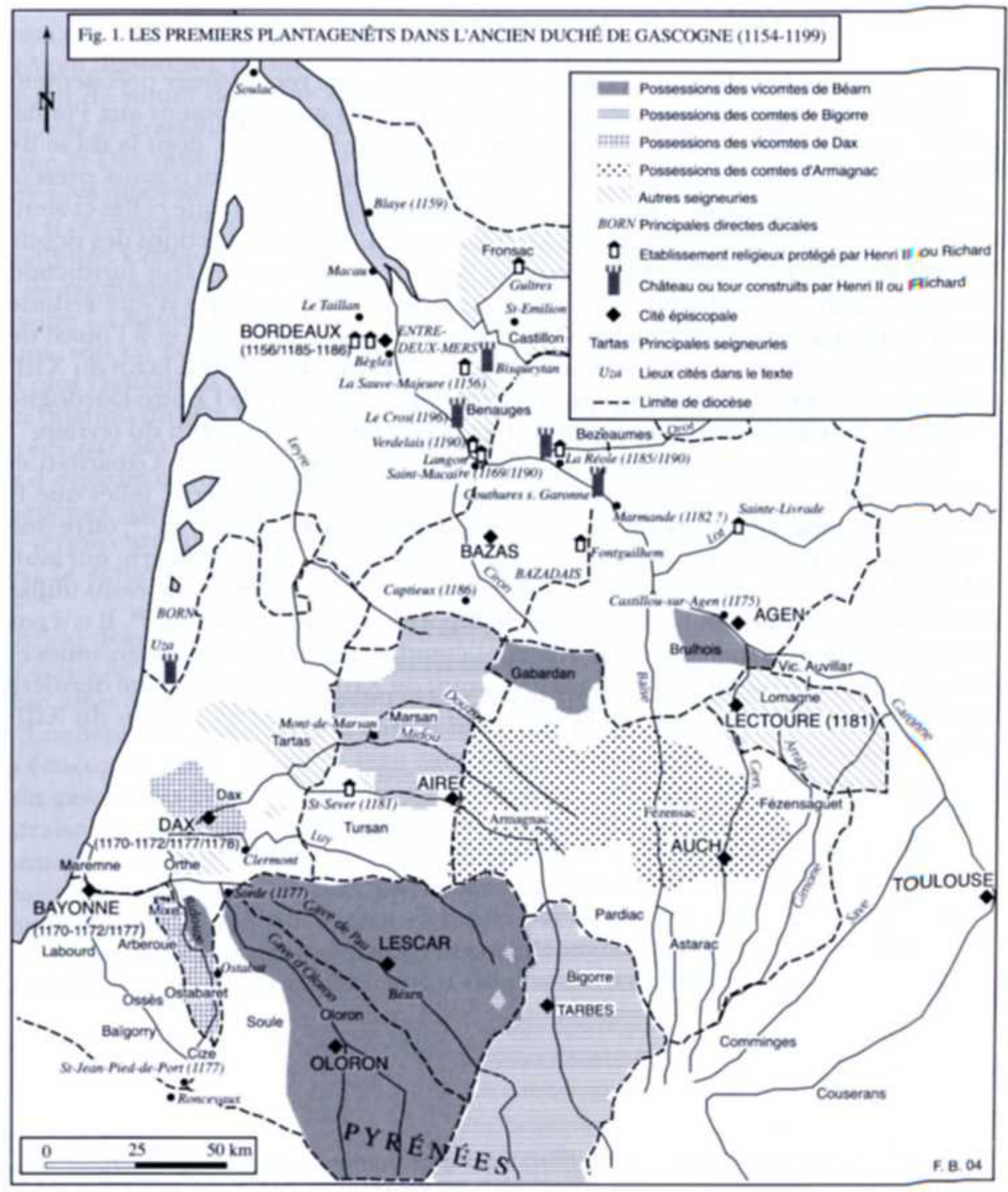


LA GASCOGNE SOUS L.ES PREMIERS PI_ANTAGENÊTS ( $\left.1154^{-1} 199\right)$

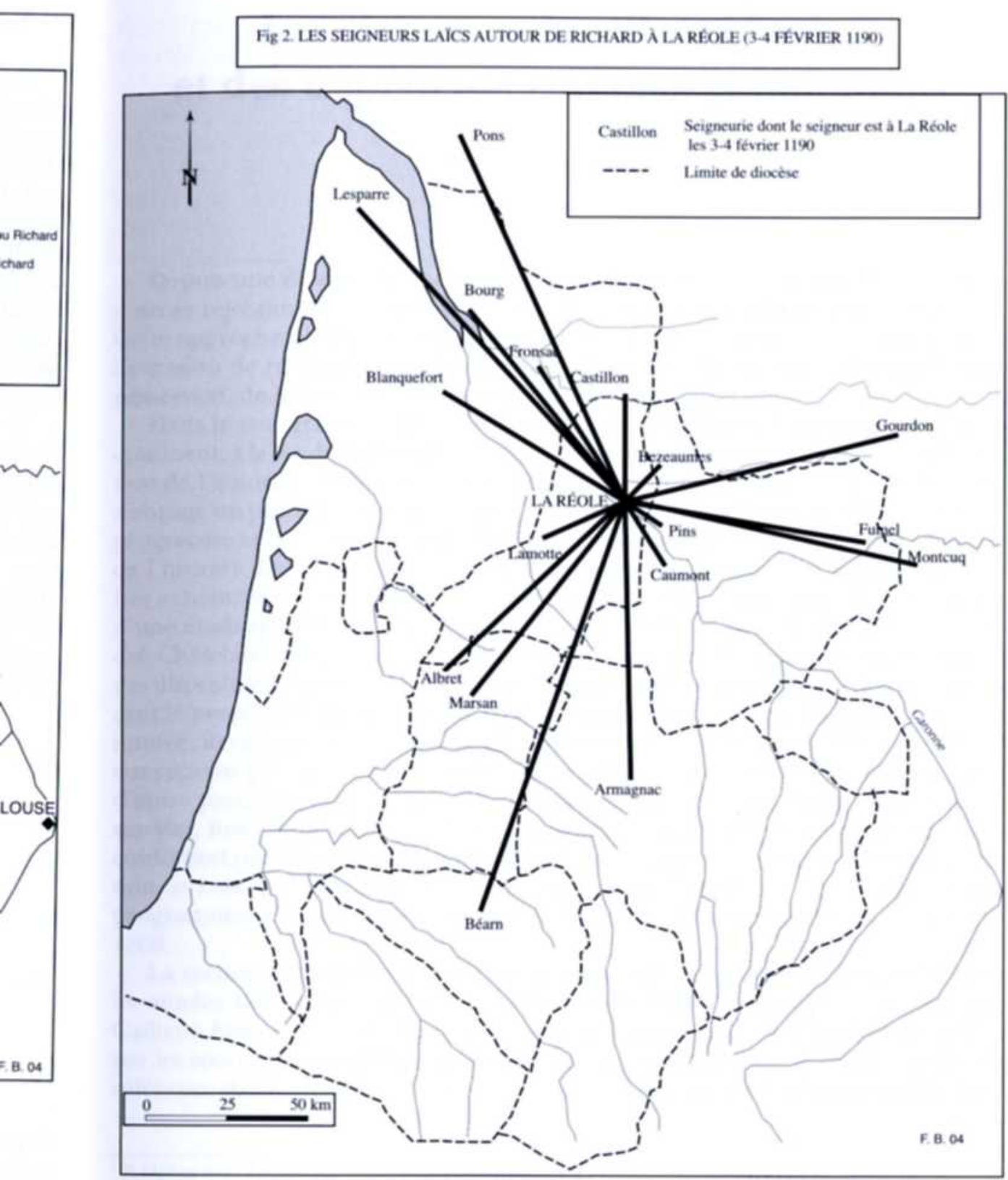

